\title{
Holographic complexity for black branes with momentum relaxation
}

\author{
Davood Mahdavian Yekta, ${ }^{1, *}$ H. Babaei-Aghbolagh, ${ }^{2, \dagger}$ Komeil Babaei Velni $\odot,{ }^{3,4, \hbar}$ and H. Mohammadzadeh ${ }^{2, \S}$ \\ ${ }^{1}$ Department of Physics, Hakim Sabzevari University, P.O. Box 397, Sabzevar, Iran \\ ${ }^{2}$ Department of Physics, University of Mohaghegh Ardabili, P.O. Box 179, Ardabil, Iran \\ ${ }^{3}$ Department of Physics, University of Guilan, P.O. Box 41335-1914, Rasht, Iran \\ ${ }^{4}$ School of Physics and School of Particles and Accelerators, Institute for Research in Fundamental \\ Sciences (IPM), P.O. Box 19395-5531, Tehran, Iran
}

(Received 12 December 2020; accepted 1 October 2021; published 21 October 2021)

\begin{abstract}
We employ the "complexity equals action" conjecture to investigate the action growth rate for the charged and neutral AdS black branes of a holographic toy model consisting of Einstein-Maxwell theory in $(d+1)$-dimensional bulk spacetime with $d-1$ massless scalar fields, which is called Einstein-Maxwellaxion theory. From the holographic point of view, the scalar fields source a spatially dependent field theory with momentum relaxation on the boundary, which is dual to the homogeneous and isotropic black branes. We find that the growth rate of the holographic complexity within the Wheeler-DeWitt patch saturates the corresponding Lloyd bound at the late-time limit. Especially for the neutral AdS black branes, it will be shown that the complexity growth rate at late time vanishes for a particular value of the relaxation parameter $\beta_{\max }$ where the temperature of the black hole is minimal. Then, we investigate the transport properties of the holographic dual theory in the minimum temperature. A nonlinear contribution of the axion field kinetic term in the context of the $k$-essence model in the four-dimensional spacetime is considered as well. We also study the time evolution of the holographic complexity for the dyonic AdS black branes in this model.
\end{abstract}

DOI: 10.1103/PhysRevD.104.086025

\section{INTRODUCTION}

The AdS/CFT correspondence [1-3], as the most important realization of the holographic principle [4,5], relates a gravity theory in an asymptotically anti-de Sitter (AdS) spacetime in the bulk to a conformal field theory (CFT) without gravity living on the boundary of this spacetime. It suggests nontrivial connections between different areas of physics - in particular, between general relativity and quantum information theory. One of the outstanding developments in this correspondence is the seminal work of Ryu and Takayanagi [6,7], which provides a holographic dictionary for the calculation of the entanglement entropy of the boundary theory. According to this proposal, the entanglement entropy of the boundary theory is equivalent to the area of a certain minimal surface in the bulk geometry. In other words, the dynamics of the bulk spacetime emerges from the quantum entanglement of the boundary theory [8]. However, the entanglement entropy

\footnotetext{
*d.mahdavian@hsu.ac.ir

†h.babaei@uma.ac.ir

*babaeivelni@guilan.ac.ir

§mohammadzadeh@uma.ac.ir
}

Published by the American Physical Society under the terms of the Creative Commons Attribution 4.0 International license. Further distribution of this work must maintain attribution to the author(s) and the published article's title, journal citation, and DOI. Funded by SCOAP. may not be enough to probe the degrees of freedom in black hole interiors, since the volume of a black hole continues growing even if spacetimes reach thermal equilibrium [9]. It is believed that quantum complexity is the correct quantity, which can continue to grow even after reaching thermal equilibrium, similar to the growth of black hole interiors.

In the framework of quantum information theory, quantum complexity is defined by the minimal number of quantum gates needed to build a target state from a reference state $[10,11]$. However, the AdS/CFT correspondence provides two proposals to compute the complexity of states in the boundary quantum field theory of two-sided AdS black holes. The first one is the "complexity = volume" $(\mathrm{CV})$ conjecture, which assumes that the quantum complexity of the CFT on the boundary is dual to the maximum volume of the Einstein-Rosen bridge-i.e., $V$-in the bulk spacetime $[12,13]$ :

$$
\mathcal{C}_{V} \sim \frac{V}{G \ell_{\text {AdS }}}
$$

where $\ell_{\text {AdS }}$ is the radius of curvature of the AdS spacetime and $G$ is the Newton constant. The second proposal is the "complexity = action" (CA) conjecture, which states that the quantum complexity on the boundary is associated with the gravitational action evaluated on a region of the WheelerDeWitt (WDW) patch in the bulk spacetime $[14,15]$ : 


$$
\mathcal{C}_{A} \sim \frac{I_{\mathrm{WDW}}}{\pi \hbar} .
$$

Moreover, when the WDW patch, besides space- or timelike boundaries, includes null boundary surfaces [16] which can join with each other, the strategy of the CA conjecture has been suggested in Ref. [17].

In general, holographic complexity has been suggested in Ref. [18] for the eternal two-sided AdS black holes on the gravity side. From the field theory point of view, this geometry is dual to a thermofield double state as follows:

$$
\left|\psi_{\mathrm{TFD}}\right\rangle=\frac{1}{\sqrt{Z}} \sum_{j} e^{-E_{j} /(2 T)} e^{-i E_{j}\left(t_{L}+t_{R}\right)}\left|E_{j}\right\rangle_{L}\left|E_{j}\right\rangle_{R},
$$

where $L$ and $R$ refer to the two copies of the boundary CFTs. The entanglement between the $L$ and $R$ copies is due to the Einstein-Rosen bridge that connects two regions. Since the complexity is conjectured to grow with time and this property is also shared with the Einstein-Rosen bridge, in Refs. [18,19], it was conjectured that the complexity could be identified with the volume of the maximal codimension-1 surface that ends to the boundary times $t_{L}$ and $t_{R}$.

The growth rate of the holographic complexity is one of the noticeable outcomes in the CA conjecture that asserts the late-time growth rate is proportional to $2 M / \pi$, independent of the boundary curvature and the spacetime dimensions $[14,15]$. It was also suggested that this quantity has an upper bound which is proportional to the total energy of the system:

$$
\dot{\mathcal{C}} \leq \frac{2 E}{\pi \hbar},
$$

where this inequality is known as the Lloyd bound [20] derived from the Margolus-Levitin theorem [21] under the assumption that each gate will evolve from a generic state into an orthogonal state. In the gravitational picture, the mass of the black hole, $M$, is regarded as the energy, $E$. The generalizations of this bound for charged and rotating black holes, respectively, are given in Refs. [15,22] as follows:

$$
\begin{aligned}
& \frac{d I_{\mathrm{WDW}}}{d t} \leq 2\left[(M-\mu Q)-(M-\mu Q)_{g s}\right], \\
& \frac{d I_{\mathrm{WDW}}}{d t} \leq 2\left[(M-\Omega J)-(M-\Omega J)_{g s}\right],
\end{aligned}
$$

where $\mu$ and $\Omega$ are the chemical potential and angular velocity of the black holes, respectively. $Q$ and $J$ are the black hole charge and angular momentum, respectively. Intuitively, these conserved charges impose a tighter bound because they provide a barrier to the rapid complexification, and consequently, some energy is tied up in noncomputing degrees of freedom. The subscript $g s$ denotes the ground state of the black hole. However, it is known
$[14,20]$ that this proposal is violated at least at early times in holographic theories [23-26], and in sufficiently exotic computational setups in nonholographic theories as well [27-29]. The late-time violation of this bound has been considered for holographic models in Refs. [25,26,30-34]. Different aspects of holographic complexity such as subregion complexity [35-38], UV divergencies of complexity [22,39-42], higher-derivative gravities [34,43-48], and Einstein-Maxwell-dilaton gravity $[25,26,49,50]$ have been studied in both the CV and CA conjectures. Attempts to define the complexity more rigorously in quantum field theory and in a continuous way, where interestingly their results in different setups match with results from holography, can be found in Refs. [51-57].

Holographic correspondence has also provided us a powerful tool to study the behavior of strongly correlated materials in condensed matter (CM) physics [58-60], which can be mapped to the classical bulk gravity. Especially, much attention has been paid to the holographic description of systems with momentum relaxation. Such systems with broken translational symmetry are needed to give a realistic description of materials in many $\mathrm{CM}$ systems [61-66]. Since momentum is conserved in a system with translational symmetry, a constant electric field can generate a charge current without current dissipation in the presence of a nonzero charge density. Thus, the conductivity of the system would become divergent at zero frequency. In more realistic CM materials, the momentum is not conserved due to impurities or a lattice structure, leading to a finite dc conductivity. There are various ways to achieve momentum dissipation, such as periodic potentials, lattices, and breaking diffeomorphism invariance [67-74]. However, there are two well-known strategies to produce momentum dissipation by the inclusion of matter fields that break the translational invariance in the dual field theory: the case of scalar fields [EMA (Einstein-Maxwell-axion) theory] that linearly depend on the horizon coordinates as given in Ref. [72], and the case of massive gravity theories which present a broken diffeomorphism invariance in the bulk as done in Ref. [75].

As the main purpose of this paper, we employ the CA conjecture to study holographic complexity and its time evolution in EMA theory with momentum relaxation by following the approach used in Ref. [23]. In particular, we compute these quantities for the charged and neutral $\mathrm{AdS}_{d+1}$ black branes and investigate the Lloyd bound for these solutions. Though one can also employ the CV conjecture to study the evolution of the holographic complexity, it has some unsatisfactory elements that make $\mathrm{CA}$ a more interesting option. For instance, in the $\mathrm{CV}$ picture, we need to introduce an arbitrary length scale by hand, while in CA this is not necessary; also, in the CV picture one must find the volume of a maximal slice in the bulk, while CA associates with the boundary state on the entire WDW patch and is easier to work with than a special 
maximal volume. The CA conjecture can also satisfy the Lloyd complexity growth bound in very general cases $[14,15]$. In fact, CA inherits all the nice features of CV duality and none of the unsatisfactory elements.

We provide an analytical discussion for the effects of the strength of momentum relaxation on the desired quantities. The results show that the Lloyd bound is saturated only at the late-time limit, and there is an upper bound on the strength of the momentum relaxation in each sector, which provides a minimum temperature for the gravitational system to have positive energy. This specific value of the relaxation parameter also motivates us to investigate the characteristic properties of the strongly correlated materials in CM physics [76]. In fact, we study the thermal conductivity and diffusivity of strongly coupled theories which are holographically dual to the EMA theory [77-79]. Inspired by the strange metals characterized by a minimum Planckian relaxation timescale $\tau_{L}$, it has been proposed in Ref. [76] that there is a universal bound for the diffusivities in the incoherent limit $\mathcal{D}_{T} \geq v_{B}^{2} \tau_{L}$, where $v_{B}$ is a characteristic velocity of the so-called butterfly velocity [78]. We will show that the diffusion constant in the EMA theory saturates this bound in the corresponding minimum temperature. In addition, we examine the effect of the nonlinear contribution of the scalar field kinetic term [8082] on the complexity growth rate in four-dimensional spacetime. This theory is known as the $k$-essence model of dark energy [80], in which the acceleration of the Universe (both at early and late times) can be driven by the kinetic energy instead of the potential energy of the scalar field. The time evolution of the holographic entanglement entropy and complexity under a thermal quench has been recently studied for EMA theory in Ref. [83], in the context of the CV conjecture.

The structure of this paper is organized as follows: in Sec. II, we review the EMA theory with momentum relaxation and study the time evolution of the holographic complexity for charged/neutral $\mathrm{AdS}_{d+1}$ black branes. In the context of the CA conjecture, we consider the WDW patch that includes null sheets bounding the bulk and joint terms, and investigate how the holographic complexity approaches the late-time limit on them. We will also investigate the transport properties of the dual theory in CM physics from a holographic point of view. In Sec. III, we study the dyonic AdS black branes in the presence of the nonlinear kinetic term in the $k$-essence model. In calculating the growth rate, the contribution of the Maxwell surface term to the action will be considered, as well. Finally, Sec. IV is dedicated to a brief summary and concluding remarks.

\section{EMA THEORY WITH MOMENTUM RELAXATION}

In order to have momentum relaxation and finite conductivity, it is essential to construct holographic models with broken translational symmetry. Thus, we consider a model in which the Einstein-Maxwell action in $(d+1)$ dimensional spacetime is supplemented by $d-1$ massless scalar fields that break the translational invariance of the boundary theory in the context of the AdS/CFT duality [72]. This theory is known as the EMA theory in Horndeski theories of modified gravity $[84,85]$. Scalar axion fields enter the bulk action only through the kinetic term $\partial_{\mu} \psi_{I}$, and the sources are linear in the boundary-i.e., $\psi_{I}^{(0)} \propto \beta_{I i} x^{i}$, where $\beta$ represents the strength of the momentum relaxation.

The action of this holographic model in the bulk is described by Ref. [72]

$$
\begin{aligned}
I_{\text {bulk }}= & \frac{1}{16 \pi G} \int_{M} d^{d+1} x \sqrt{-g}[R-2 \Lambda \\
& \left.-\frac{1}{4} F_{\mu \nu} F^{\mu \nu}-\frac{1}{2} \sum_{I}^{d-1}\left(\partial \psi_{I}\right)^{2}\right]
\end{aligned}
$$

where $G$ is a $(d+1)$-dimensional gravitational constant and $\Lambda=-d(d-1) / 2 L^{2}$ is a cosmological term. The action includes the field strength $F_{\mu \nu}=\partial_{\mu} A_{\nu}-\partial_{\nu} A_{\mu}$ of a $U(1)$ gauge field $A_{\mu}$ and $d-1$ massless scalar fields $\psi_{I}$. The model admits the homogeneous and isotropic charged $\operatorname{AdS}_{d+1}$ black brane solutions of radius $L$ with nontrivial scalar field sources. They are described by the following ansatz:

$$
\begin{gathered}
d s^{2}=-f(r) d t^{2}+\frac{d r^{2}}{f(r)}+r^{2} \delta_{i j} d x^{i} d x^{j}, \\
A=A_{t}(r) d t, \quad \psi_{I}=\beta_{I i} x^{i},
\end{gathered}
$$

where $i$ labels the $d-1$ spatial $x_{i}$ directions and $I$ is an internal index that labels the $d-1$ scalar fields. Substituting ansatz (2.2) into the equations of motion derived from the action (2.1), we find that

$$
\begin{aligned}
& f(r)=r^{2}-\frac{\beta^{2}}{2(d-2)}-\frac{m_{0}}{r^{d-2}}+\frac{q^{2}}{r^{2(d-2)}}, \\
& A_{t}(r)=\sqrt{\frac{2(d-1)}{d-2}} q\left(\frac{1}{r_{h}^{d-2}}-\frac{1}{r^{d-2}}\right),
\end{aligned}
$$

where

$$
\begin{aligned}
\beta^{2} & \equiv \frac{1}{d-1} \sum_{i}^{d-1} \vec{\beta}_{i} \cdot \vec{\beta}_{i}, \\
\vec{\beta}_{i} \cdot \vec{\beta}_{j} & =\sum_{I} \beta_{I i} \beta_{I j}=\beta^{2} \delta_{i j} \quad \forall i, j .
\end{aligned}
$$

Note that for the AdS radius, we set $L=1$ in the rest of the paper.

The mass parameter $m_{0}$, which is proportional to the energy density of the brane, is computed from $f\left(r_{h}\right)=0$ as follows: 


$$
m_{0}=r_{h}^{d}\left(1+\frac{q^{2}}{r_{h}^{2(d-1)}}-\frac{1}{2(d-2)} \frac{\beta^{2}}{r_{h}^{2}}\right)
$$

where $r_{h}$ is the location of the event horizon. This is related to the mass of the brane with Ref. [86]

$$
M=\frac{(d-1) V_{d-1}}{16 \pi G} m_{0}
$$

Here, $V_{d-1}$ is the dimensionless volume of the relevant spatial geometry. The parameter $q$ is related to the charge of the brane through Gauss's law with

$$
Q=\frac{\sqrt{2(d-1)(d-2)} V_{d-1}}{16 \pi G} q
$$

The Hawking temperature and the entropy of the branes are given by

$T=\frac{f^{\prime}\left(r_{h}\right)}{4 \pi}=\frac{1}{4 \pi}\left(d r_{+}-\frac{\beta^{2}}{2 r_{h}}-\frac{(d-2) q^{2}}{r_{h}^{d-1}}\right)$,

$S=\frac{V_{d-1}}{16 \pi G} 4 \pi r_{h}^{(d-1)}$.

Since the blackening factor in Eq. (2.3) has two real roots, $r_{+}$and $r_{-}$(where $r_{+}>r_{-}$), corresponding to the outer and inner horizons in which $f\left(r_{+}\right)=f\left(r_{-}\right)=0$, we can define a chemical potential for both of them as follows:

$$
\begin{gathered}
\mu_{+}=\left.\frac{\partial M}{\partial Q}\right|_{V, S}=\sqrt{\frac{2(d-1)}{d-2}} \frac{q}{r_{+}^{d-2}}, \\
\mu_{-}=\left.\frac{\partial M}{\partial Q}\right|_{V, S}=\sqrt{\frac{2(d-1)}{d-2}} \frac{q}{r_{-}^{d-2}} .
\end{gathered}
$$

Various features of the thermodynamics of this solution have been extensively studied in Ref. [87].

\section{A. Complexity of charged black branes via the $\mathrm{CA}$ conjecture}

We use the CA conjecture [Eq. (1.2)] to compute the holographic complexity for the charged $\mathrm{AdS}_{d+1}$ black branes in the EMA theory. The essential ingredient in this method is to evaluate the action on a WDW patch $[14,15]$. However, we follow the method of Ref. [23], in which the action in the WDW patch includes not only the bulk theory and the Gibbons-Hawking-York (GHY) boundary term $[88,89]$, but also boundary segments of joint terms due to the intersection of the timelike, spacelike, and null boundaries [16]. This will be the general strategy followed in the rest of the paper. The contribution of the GHY surface terms is
$I_{\text {bdy }}=\frac{1}{8 \pi G} \int_{\mathcal{B}} d^{d} x \sqrt{\gamma} K-\frac{1}{8 \pi G} \int_{\mathcal{B}^{\prime}} d \lambda d^{d-1} \theta \sqrt{\gamma} \kappa$,

where $K$ is the trace of the extrinsic curvature $K_{\mu \nu}=-\gamma_{\mu}^{\rho} \gamma_{\nu}^{\sigma} \nabla_{(\rho} n_{\sigma)}, \gamma_{\mu \nu}$ is the induced metric on the boundary, and $n_{\mu}$ is the outward-pointing unit normal vector to the boundary. $\kappa$ is the surface term for the null segments, which measures the failure of the null generators to be affinely parametrized and which is assumed to vanish-i.e., it does not have any contribution to CA for null segments.

The joint actions are given by

$I_{\text {joint }}=\frac{1}{8 \pi G} \int_{\Sigma} d^{d-1} x \sqrt{\sigma} \varrho+\frac{1}{8 \pi G} \int_{\Sigma^{\prime}} d^{d-1} x \sqrt{\sigma} a$,

in which $Q$ appears when we have the intersection of timelike or spacelike boundaries, the so-called Hayward terms [16], while $a$ is required when one or both of the intersecting boundaries belong to null surfaces [17]. The general rules for the construction of the former joint terms could also be found in Refs. [17,36]. In particular, for timelike normals $\mathbf{t}_{i}$, spacelike normals $\mathbf{n}_{i}$ and auxiliary unit vectors $\hat{\mathbf{t}}_{i}$ and $\hat{\mathbf{n}}_{i}, \varrho$ is given by

$\varrho=\operatorname{arccosh}\left|\mathbf{t}_{1} \cdot \mathbf{t}_{2}\right| \operatorname{sign}(\varrho)=-\operatorname{sign}\left(\mathbf{t}_{1} \cdot \mathbf{t}_{2}\right) \operatorname{sign}\left(\hat{\mathbf{n}}_{1} \cdot \mathbf{t}_{2}\right)$,

$\varrho=\operatorname{arccosh}\left|\mathbf{n}_{1} \cdot \mathbf{n}_{2}\right| \operatorname{sign}(\varrho)=-\operatorname{sign}\left(\mathbf{n}_{1} \cdot \mathbf{n}_{2}\right) \operatorname{sign}\left(\mathbf{n}_{1} \cdot \hat{\mathbf{t}}_{2}\right)$,

$\varrho=\operatorname{arcsinh}\left|\epsilon \mathbf{t}_{1} \cdot \mathbf{n}_{2}\right| \epsilon=-\operatorname{sign}\left(\mathbf{n}_{2} \cdot \hat{\mathbf{n}}_{1}\right)$.

However, these are not relevant here, since all of the joints that we consider in the WDW patches involve at least one null surface. The latter ones are also defined appropriately in the next subsection. There is also a counterterm action for the null surfaces as

$$
I_{\mathrm{ct}}=\frac{1}{8 \pi G} \int_{\mathcal{B}^{\prime}} d \lambda d^{d-1} \theta \sqrt{\gamma} \Theta \log \left(\ell_{c} \Theta\right),
$$

which is introduced to ensure reparametrization invariance on the null boundaries. $\Theta$ is the expansion parameter that is related to the induced metric as

$$
\Theta=\partial_{\lambda} \log \sqrt{\gamma}
$$

and $\ell_{c}$ is an arbitrary length scale. A precise definition of parameters, boundary metrics, and comprehensive discussions of these actions are given in Ref. [17]. In brief, the total action is defined by

$$
I_{\mathrm{tot}}=I_{\mathrm{bulk}}+I_{\mathrm{bdy}}+I_{\mathrm{joint}}+I_{\mathrm{ct}}
$$

Of course, there may be a boundary term for the Maxwell field in this action that does not change the equations of motion, but it affects the variational principle for the 
Maxwell field, and one should change the boundary conditions consistently [90]. We will consider the contribution of such a term for charged geometries in the next section.

Due to the presence of null boundaries in the total action, it is more convenient to introduce the ingoing and outgoing coordinates

$$
v=t+r^{*}(r), \quad u=t-r^{*}(r),
$$

where $r^{*}$ is a tortoise coordinate defined as

$$
r^{*}(r)=\int \frac{d r}{f(r)}
$$

with asymptotic behavior

$$
\lim _{r \rightarrow \infty} r^{*}(r)=r_{\infty}^{*}
$$

WDW patch: In order to study the evolution of complexity for the action (2.15), we draw the Penrose diagram of the causal structure of the charged AdS black brane described by Eq. (2.3) in Fig. 1. Following Ref. [23], the corresponding WDW patch is denoted by the shaded

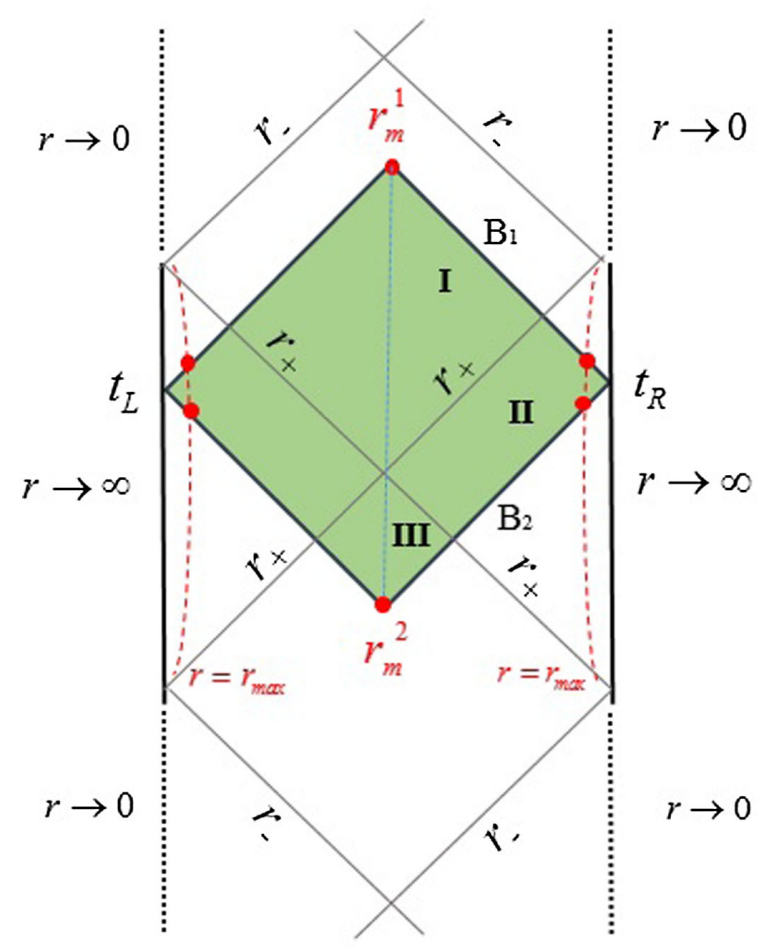

FIG. 1. Penrose diagram of the WDW patch for a charged AdS black brane in symmetric configuration $\left(t_{L}=t_{R}\right) . r \rightarrow 0$ is the singular surface, and $r \rightarrow \infty$ is the asymptotic boundary surface. The red dashed lines correspond to UV cutoff surfaces at $r=r_{\max }$, and $r_{m}^{1}, r_{m}^{2}$ are the meeting points of null boundaries in the bulk. region, which is bounded by the light sheets sent from the two asymptotic time slices $t_{L}$ and $t_{R}$. Without loss of generality, we choose the symmetric configuration for the time slices-i.e., $t_{L}=t_{R} \equiv t / 2$. In the next subsection, we evaluate the gravitational action on this patch as the boundary time increases.

The patch includes two UV cutoff surfaces near the asymptotic boundary regions at $r=r_{\max }$ which are denoted by red dashed lines in Fig. 1. In fact, the null boundaries of the WDW patch begin from the UV cutoff surface at $r=$ $r_{\max }$ and go through the bulk spacetime. There are two meeting points in the bulk which come from the intersecting future boundaries at $r=r_{m}^{1}$ and past boundaries at $r=r_{m}^{2}$. The time evolution of the WDW patch can be encoded in the time dependence of these points. These satisfy the following relations:

$\frac{t}{2}+r_{\infty}^{*}-r^{*}\left(r_{m}^{1}\right)=0, \quad \frac{t}{2}-r_{\infty}^{*}+r^{*}\left(r_{m}^{2}\right)=0$,

in which, by using Eq. (2.17), their time evolution is given by

$$
\frac{d r_{m}^{1}}{d t}=\frac{f\left(r_{m}^{1}\right)}{2}, \quad \frac{d r_{m}^{2}}{d t}=-\frac{f\left(r_{m}^{2}\right)}{2} .
$$

The null boundaries of the right sector of the corresponding WDW patch are

$B_{1}: \frac{t}{2}=r^{*}(r)-r_{\infty}^{*}, \quad B_{2}:-\frac{t}{2}=r^{*}(r)-r_{\infty}^{*}$.

These equations are important in the study of the time evolution of the total action [Eq. (2.15)].

From a holographic point of view [58], it has been proposed in Ref. [23] that this black hole geometry with $U(1)$ symmetry is dual to a charged thermofield double state:

$$
\begin{aligned}
\left|\psi_{\mathrm{CTFD}}\right\rangle= & \frac{1}{\sqrt{Z}} \sum_{j, l} e^{-E_{j}-\mu Q_{l} /(2 T)} e^{-i E_{j}\left(t_{L}+t_{R}\right)} \\
& \times\left|E_{j},-Q_{l}\right\rangle_{L}\left|E_{j}, Q_{l}\right\rangle_{R},
\end{aligned}
$$

where, comparing with the state in Eq. (1.3), in addition to the temperature $T$, this state has a chemical potential $\mu$ and electric charge $Q$. The density matrix of the corresponding grand canonical ensemble characterized by $T$ and $\mu$ is obtained by tracing out the states in its boundary.

\section{The growth rate of complexity}

In the following, we compute the growth rate of the holographic complexity on the WDW patch associated with a charged AdS black brane-see Fig. 1. In this regard, we consider the time dependence of the total action in Eq. (2.15). In the symmetric configuration of the WDW 
patch, we can perform the calculations only for the right side of the Penrose diagram — or regions I, II, and III as depicted in Fig. 1 -and then multiply the result by a factor of 2 .

Bulk action.-The Ricci scalar tensor and the kinetic terms of axion fields in this background are given by

$$
\begin{aligned}
R & =-d(d+1)+\frac{(d-1) \beta^{2}}{2 r^{2}}, \\
-\frac{1}{2} \sum_{I}^{d-1}\left(\partial \psi_{I}\right)^{2} & =-\frac{(d-1) \beta^{2}}{2 r^{2}},
\end{aligned}
$$

where the contribution of the $\beta^{2}$ term in the scalar action is canceled by its contribution from the Einstein-Hilbert action; thus, from the action (2.1) we have

$$
\begin{aligned}
I_{\text {bulk }}= & 2\left(I_{\text {bulk }}^{\mathrm{I}}+I_{\text {bulk }}^{\mathrm{II}}+I_{\text {bulk }}^{\mathrm{III}}\right) \\
= & \frac{V_{d-1}}{8 \pi G}\left[\int_{r_{m}^{1}}^{r_{+}}\left(\frac{t}{2}+r_{\infty}^{*}-r^{*}(r)\right)+2 \int_{r_{+}}^{r_{\max }}\left(r_{\infty}^{*}-r^{*}(r)\right)\right. \\
& \left.+\int_{r_{m}^{2}}^{r_{+}}\left(-\frac{t}{2}+r_{\infty}^{*}-r^{*}(r)\right)\right] I(r) d r \\
= & I_{\text {bulk }}^{0}+I_{\text {bulk }}(t)
\end{aligned}
$$

where the integrand $I(r)$ is

$$
I(r)=r^{d-1}\left[-2 d+\frac{(d-2) q^{2}}{2 r^{2(d-1)}}\right],
$$

and $I_{\text {bulk }}^{0}$ is the time-independent part of the bulk action, and only the first and third terms depend on the time through Eq. (2.19).

Boundary surface action. - If we choose affine parametrization for the null normals, then the null surface term vanishes $(\kappa=0)$; thus, we only need to consider the GHY term coming from the surface at UV cutoff on the right side of the WDW patch. The trace of the extrinsic curvature for ansatz (2.2) is given by

$$
K=\frac{1}{2}\left(\frac{\partial_{r} f(r)}{\sqrt{f(r)}}+\frac{2(d-1)}{r} \sqrt{f(r)}\right) .
$$

Following Ref. [23], we define future-directed normal vectors to evaluate $K$ :

$$
\begin{aligned}
& r=\epsilon: \quad \mathbf{t}=t_{\mu} d x^{\mu}=-\frac{d r}{\sqrt{-f(\epsilon)}}, \\
& r=r_{\max }: \quad \mathbf{s}=s_{\mu} d x^{\mu}=\frac{d r}{\sqrt{f\left(r_{\max }\right)}} .
\end{aligned}
$$

Therefore, we obtain the contribution of the surface action in Eq. (2.10) for the charged AdS solution [Eq. (2.3)] as

$$
\begin{aligned}
I_{\mathrm{bdy}}= & 2 I_{\mathrm{bdy}}^{r=r_{\text {max }}}=\frac{r^{d-1} V_{d-1}}{8 \pi G}\left(\partial_{r} f(r)+\frac{2(d-1)}{r} f(r)\right) \\
& \times\left.\left(r_{\infty}^{*}-r^{*}(r)\right)\right|_{r=r_{\text {max }}} .
\end{aligned}
$$

As is obvious, the cutoff term at $r=r_{\max }$ is independent of the time, so the boundary term has no contribution to the time evolution of the holographic complexity.

Joint action.-According to the WDW patch in Fig. 1, there are different joint contributions at the intersection of null boundaries with the surfaces at $r=r_{\max }$ and with each other at $r_{m}^{1}$ and $r_{m}^{2}$. It has been shown in Ref. [39] that the null joint contributions at the UV cutoff surfaces have no time dependence, so we need only to consider the last two joining points. Assume that $k_{1}$ and $k_{2}$ are the null vectors associated with two past null boundaries intersecting at $r_{m}^{2}$ which are defined by

$K_{1}=\xi\left(-d t+\frac{d r}{f(r)}\right), \quad K_{2}=\xi\left(d t+\frac{d r}{f(r)}\right)$,

where $\xi$ is a normalization constant for null vectors. Those for null vectors associated with two future null boundaries (intersecting at $r_{m}^{1}$ ), i.e., $\tilde{k}_{1}$ and $\tilde{k}_{2}$, are similar. Following Ref. [17], the joint term is defined by $a=\ln \left|-\frac{1}{2} k_{1} \cdot k_{2}\right|$ for the first set and by $\tilde{a}=\ln \left|-\frac{1}{2} \tilde{k}_{1} \cdot \tilde{k}_{2}\right|$ for the second set; then, the joint action (2.11) can be evaluated as

$I_{\text {joint }}=-\frac{V_{d-1}}{8 \pi G}\left[\left(r_{m}^{1}\right)^{d-1} \log \frac{\left|f\left(r_{m}^{1}\right)\right|}{\xi^{2}}+\left(r_{m}^{2}\right)^{d-1} \log \frac{\left|f\left(r_{m}^{2}\right)\right|}{\xi^{2}}\right]$,

where the time dependence of this contribution comes from Eq. (2.20).

Counterterm action.-In order to remove the ambiguity associated with the normalization of the null vectors, we need to add this boundary term to the action. Thus, we define the affine parameter $\lambda=r / \xi$ such that the total action with the counterterm does not depend on the parametrization of the null surfaces. In this parametrization, the expansion [Eq. (2.14)] takes the form Ref. [23]

$$
\Theta=\frac{(d-1) \xi}{r} .
$$

Therefore, the counterterm action in Eq. (2.13) becomes

$$
\begin{aligned}
I_{\mathrm{ct}}= & 2\left(I_{\mathrm{ct}}^{\mathrm{future}}+I_{\mathrm{ct}}^{\mathrm{past}}\right)=\frac{(d-1) V_{d-1}}{4 \pi G} \\
& \times\left(\int_{r_{m}^{1}}^{r_{\max }} r^{d-2} \log \frac{(d-1) \ell_{c} \xi}{r} d r\right. \\
& \left.+\int_{r_{m}^{2}}^{r_{\max }^{2}} r^{d-2} \log \frac{(d-1) \ell_{c} \xi}{r} d r\right) .
\end{aligned}
$$


Again, this contribution depends on the time through Eq. (2.20).

\section{The late-time behavior}

Now, we can determine the rate of change of the holographic complexity by considering all of the above contributions:

$$
\frac{d \mathcal{C}_{A}}{d t}=\frac{d}{d t}\left(\delta I_{\text {bulk }}+I_{\text {joint }}+I_{\mathrm{ct}}\right),
$$

where $\delta I_{\text {bulk }} \equiv I_{\text {bulk }}-I_{\text {bulk }}^{0}$. Henceforth, we will set $\pi \hbar=1$ in the CA conjecture in Eq. (1.2) for simplicity. Thus, the growth rate of the holographic complexity yields

$\frac{d \mathcal{C}_{A}}{d t}=\frac{(d-1) V_{d-1}}{16 \pi G}\left[\frac{2 q^{2}}{r^{d-2}}-r^{d-2} f(r) \log \frac{(d-1)^{2} \ell_{c}^{2}|f(r)|}{r^{2}}\right]_{r_{m}^{2}}^{r_{m}^{1}}$.

At late times, the future (past) corner approaches the inner (outer) horizon, such that due to the conditions $f\left(r_{+}\right)=$ $f\left(r_{-}\right)=0$, the second term vanishes. This leaves the result

$$
\begin{aligned}
\left.\frac{d \mathcal{C}_{A}}{d t}\right|_{t \rightarrow \infty} & =\frac{(d-1) V_{d-1}}{16 \pi G}\left[\frac{2 q^{2}}{r^{d-2}}\right]_{r=r_{+}}^{r=r_{-}} \\
& =\left(M-\mu_{+} Q\right)-\left(M-\mu_{-} Q\right),
\end{aligned}
$$

where we have substituted from Eqs. (2.7) and (2.9). The results in this limit are consistent with the calculations in Refs. [15,22] for the charged black holes without considering the joint and the counterterm actions directly.

For the complexity growth rate of the charged AdS black brane obtained in Eq. (2.35), it seems that in the $Q \rightarrow 0$ limit it vanishes, but as we know from general charged black holes, the $Q \rightarrow 0$ limit corresponds to $r_{-} \rightarrow 0$, so we have $\mu_{-} Q \rightarrow 2 M$ while $\mu_{+} Q \rightarrow 0$. Therefore, in this limit we recover the case of neutral AdS branes for the Lloyd bound-i.e., $d \mathcal{C}_{A} / d t=2 M$.

\section{B. Complexity of neutral AdS black branes}

For the neutral black branes, it is sufficient to insert $q=0$ into ansatz (2.3). Therefore, the mass parameter $m_{0}$, which is proportional to the energy density of the brane, is computed from $f\left(r_{h}\right)=0$, where $r_{h}$ is the position of the event horizon:

$$
m_{0}=r_{h}^{d}\left(1-\frac{1}{2(d-2)} \frac{\beta^{2}}{r_{h}^{2}}\right)
$$

and this is related to the mass of the brane with

$$
M=\frac{(d-1) V_{d-1}}{16 \pi G} m_{0} .
$$

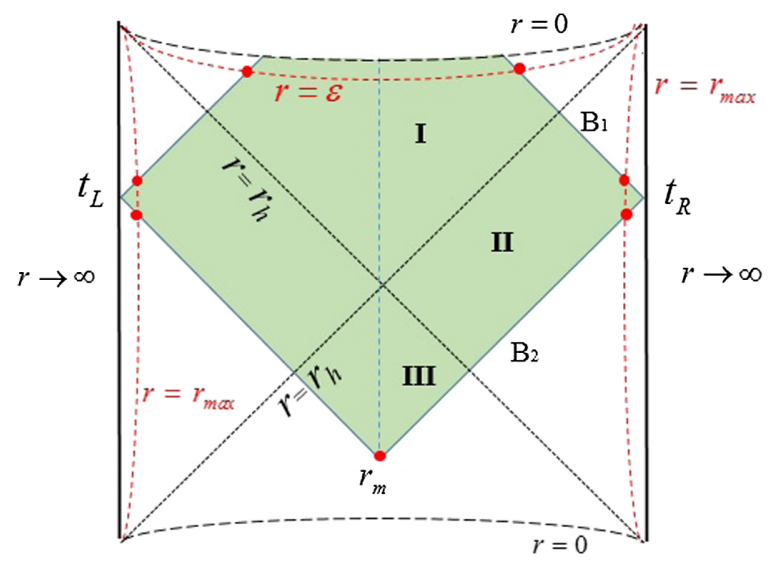

FIG. 2. Penrose diagram of the WDW patch of a neutral AdS black brane in symmetric configuration $\left(t_{L}=t_{R}\right)$.

Also, the Hawking temperature and the entropy for this solution are given by

$T=\left.\frac{f^{\prime}(r)}{4 \pi}\right|_{r=r_{h}}=\frac{2 d r_{h}^{2}-\beta^{2}}{8 \pi r_{h}}, \quad S=\frac{V_{d-1}}{16 \pi G} 4 \pi r_{h}^{(d-1)}$.

WDW patch: The causal structure of a two-sided neutral AdS black brane with a single horizon is described by the Penrose diagram in Fig. 2. The corresponding WDW patch is denoted by the shaded region bounded by the light sheets sent from the two asymptotic time slices $t_{L}$ and $t_{R}$. We choose the symmetric configuration for the time slicesi.e., $t_{L}=t_{R} \equiv t / 2$. In the next subsection, we evaluate the total action [Eq. (2.15)] on this patch as the boundary time increases.

In this patch, $r_{m}$ is the point at which the past light sheets from the left and right boundaries intersect before hitting the past singularity at some critical time $t_{c}$ in the symmetric configuration

$$
\frac{t_{c}}{2}=r_{\infty}^{*}-r^{*}(0) .
$$

It also contains a cutoff surface near the future singularity at $r=\epsilon$ and two surfaces near the asymptotic boundary regions at $r=r_{\max }$. These surfaces are specified by the dashed red lines in Fig. 2. The boundaries $B_{1}$ and $B_{2}$ are given by Eq. (2.21).

\section{The growth rate of complexity}

In the following, we compute the growth rate of the holographic complexity on the WDW patch associated with a two-sided AdS black brane for times $t>t_{c}$-see Fig. 2 . In this regard, we consider the time dependence of the total action in Eq. (2.15). In the symmetric configuration of the WDW patch, we can perform the calculations only for the 
right side of the Penrose diagram or regions I, II, and III, and then multiply the result by a factor of 2 .

Bulk action.-The bulk contribution comes from the action (2.1) by omitting the Maxwell term. Therefore, according to the relations in Eq. (2.23), for $t>t_{c}$ we have

$$
\begin{aligned}
I_{\text {bulk }}= & 2\left(I_{\text {bulk }}^{\mathrm{I}}+I_{\text {bulk }}^{\mathrm{II}}+I_{\text {bulk }}^{\mathrm{III}}\right) \\
= & -\frac{d V_{d-1}}{4 \pi G}\left[\int_{\epsilon}^{r_{h}}\left(\frac{t}{2}+r_{\infty}^{*}-r^{*}(r)\right)\right. \\
& +2 \int_{r_{h}}^{r_{\max }}\left(r_{\infty}^{*}-r^{*}(r)\right) \\
& \left.+\int_{r_{m}}^{r_{h}}\left(-\frac{t}{2}+r_{\infty}^{*}-r^{*}(r)\right)\right] r^{d-1} d r \\
= & I_{\text {bulk }}^{0}-\frac{d V_{d-1}}{4 \pi G} \int_{\epsilon}^{r_{m}}\left(\frac{t}{2}+r_{\infty}^{*}-r^{*}(r)\right) r^{d-1} d r .
\end{aligned}
$$

Clearly, only the second term depends on the time.

Boundary surface action.--If we choose affine parametrization for the null normals, then the null surface term vanishes $(\kappa=0)$; thus, we only need to consider the GHY term coming from the regulator surface at the future singularity and the surface at the UV cutoff. Therefore, we obtain the contribution of the surface action in Eq. (2.10) for the neutral AdS solution as

$$
\begin{aligned}
I_{\mathrm{bdy}}= & 2\left(I_{\mathrm{bdy}}^{r=\epsilon}+I_{\mathrm{bdy}}^{r=r_{\max }}\right) \\
= & -\frac{d V_{d-1}}{8 \pi G}\left[\left(2 r^{d}-r_{h}^{d}\right)-\frac{2(d-1) r^{d-2}-d r_{h}^{d-2}}{2 d(d-2)} \beta^{2}\right] \\
& \times\left(\left.\left(t / 2+r_{\infty}^{*}-r^{*}(r)\right)\right|_{r=\epsilon}-\left.\left(r_{\infty}^{*}-r^{*}(r)\right)\right|_{r=r_{\max }}\right) .
\end{aligned}
$$

As seen, the cutoff term at $r=r_{\max }$ is independent of the time, so we can rewrite Eq. (2.41) as

$$
\begin{aligned}
I_{\mathrm{bdy}}= & I_{\mathrm{bdy}}^{0}-\frac{d V_{d-1}}{8 \pi G}\left[\left(2 r^{d}-r_{h}^{d}\right)-\frac{2(d-1) r^{d-2}-d r_{h}^{d-2}}{2 d(d-2)} \beta^{2}\right] \\
& \times\left.\left(t / 2+r_{\infty}^{*}-r^{*}(r)\right)\right|_{r=\epsilon},
\end{aligned}
$$

where $I_{\mathrm{bdy}}^{0}$ is independent of the time and has no contribution to the growth rate.

Joint action.-According to the WDW patch in Fig. 2, there are different joint contributions at the intersection of the null boundaries with surfaces at $r=\epsilon$ and $r=r_{\max }$, and with each other at $r_{m}$. However, the joints at singular and cutoff surfaces are independent of the time. Assuming that $k_{1}$ and $k_{2}$ are given by the relations in Eq. (2.29), the joint action [Eq. (2.11)] can be evaluated as

$$
I_{\text {joint }}=-\frac{V_{d-1}}{8 \pi G}\left[r^{d-1} \log \frac{|f(r)|}{\xi^{2}}\right]_{r=r_{m}} .
$$

The time evolution of this contribution is through the implicit time dependence of $r_{m}$ with the equation

$$
\frac{t}{2}-r_{\infty}^{*}+r^{*}\left(r_{m}\right)=0 .
$$

Counterterm action.-Using the parameter expansion introduced in Eq. (2.31), the counterterm action becomes

$$
\begin{aligned}
I_{\mathrm{ct}} & =2\left(I_{\mathrm{ct}}^{\mathrm{future}}+I_{\mathrm{ct}}^{\mathrm{past}}\right) \\
& =\frac{(d-1) V_{d-1}}{4 \pi G}\left(\int_{\epsilon}^{r_{\max }}+\int_{r_{m}}^{r_{\max }}\right) r^{d-2} \log \frac{(d-1) \ell_{c} \xi}{r} d r .
\end{aligned}
$$

It is clear that the cutoff bounds have no time dependence; thus, in the limit $\epsilon \rightarrow 0$, the counterterm action depends on the time through Eq. (2.44).

\section{The late-time behavior}

In the case of neutral branes, we should also consider the contribution of boundary terms - that is, the growth rate for $t>t_{c}$ is calculated from

$$
\frac{d \mathcal{C}_{A}}{d t}=\frac{d}{d t}\left(\delta I_{\text {bulk }}+\delta I_{\text {bdy }}+I_{\text {joint }}+I_{\mathrm{ct}}\right),
$$

where $\delta I_{\text {bulk }} \equiv I_{\text {bulk }}-I_{\text {bulk }}^{0}$ and $\delta I_{\text {bdy }} \equiv I_{\text {bdy }}-I_{\text {bdy }}^{0}$. From Eqs. (2.40) and (2.42) for the bulk and boundary actions,

$$
\begin{aligned}
\frac{d I_{\text {joint }}}{d t}= & \frac{V_{d-1}}{8 \pi G}\left[r_{m}^{d}+\frac{1}{2}(d-2) r_{h}^{d}\right. \\
& +\frac{1}{2}(d-1)\left(r_{m}^{d}-r_{h}^{d}\right) \log \frac{\left|f\left(r_{m}\right)\right|}{\xi^{2}} \\
& \left.+\left(\frac{(d-1)\left(r_{h}^{d-2}-r_{m}^{d-2}\right)}{4(d-2)} \log \frac{\left|f\left(r_{m}\right)\right|}{\xi^{2}}-\frac{1}{4} r_{h}^{d-2}\right) \beta^{2}\right]
\end{aligned}
$$

for the joint term, and the following for the counterterm action:

$$
\begin{aligned}
\frac{d I_{\mathrm{ct}}}{d t}= & \frac{(d-1) V_{d-1}}{16 \pi G}\left[\left(r_{m}^{d}-r_{h}^{d}\right)-\frac{\left(r_{m}^{d-2}-r_{h}^{d-2}\right) \beta^{2}}{2(d-2)}\right] \\
& \times \log \frac{(d-1) \ell_{c} \xi}{r_{m}},
\end{aligned}
$$

we obtain the growth rate of the holographic complexity in the CA conjecture as 


$$
\begin{aligned}
\frac{d \mathcal{C}_{A}}{d t}= & \frac{(d-1) V_{d-1}}{8 \pi G}\left[r_{h}^{d}-\frac{r_{h}^{d-2} \beta^{2}}{2(d-2)}\right. \\
& \left.+\frac{1}{2} r_{m}^{d-2} f\left(r_{m}\right) \log \frac{(d-1)^{2} \ell_{c}^{2}\left|f\left(r_{m}\right)\right|}{r_{m}^{2}}\right],
\end{aligned}
$$

or equivalently, from Eq. (2.37), it can be recast in the form

$$
\begin{aligned}
\frac{d \mathcal{C}_{A}}{d t}= & 2 M+\frac{V_{d-1}}{16 \pi G}\left[(d-1) r_{m}^{d-2} f\left(r_{m}\right)\right. \\
& \left.\times \log \frac{(d-1)^{2} \ell_{c}^{2}\left|f\left(r_{m}\right)\right|}{r_{m}^{2}}\right],
\end{aligned}
$$

where the time dependence of the meeting point $r_{m}$ is obtained from Eq. (2.44) and the definition of the tortoise coordinate in Eq. (2.17) as

$$
\frac{d r_{m}}{d t}=-\frac{f\left(r_{m}\right)}{2}
$$

One can observe that the result (2.50) does not satisfy the Lloyd bound on the rate of quantum computation [20] for $t>t_{c}$, but at the late-time limit in which $r_{m}$ approaches $r_{h}$ and $f\left(r_{m}\right) \rightarrow 0$, the contribution of the second term vanishes, and this yields

$$
\left.\frac{d \mathcal{C}_{A}}{d t}\right|_{t \rightarrow \infty}=2 M
$$

which is consistent with the expected rate of growth at late time in Refs. [14,15] even in the presence of momentum relaxation. The joint and the counterterm contributions of both charged and neutral solutions are sensitive to the ambiguities of null boundaries through the normalization constant $\xi$, but according to Eqs. (2.34) and (2.49), the result for the total action is independent of that.

More specifically, the relation (2.36) yields a bound on the value of $\beta$ such that a larger value of $\beta$ leads to a negative value for the mass, which has no physical interpretation. For the neutral branes studied in this section, this maximum value is obtained from $\beta_{\max }^{2}=2(d-2) r_{h}^{2}$. We investigate that for $\beta \leq \beta_{\max }$, the equation $f(r)=0$ always has a real positive root (single horizon $r_{h}$ ) even when $m_{0}=0$. Further, from the definition of temperature in Eq. (2.38), this value then yields a finite temperature $T=r_{h} / 2 \pi$ in this case. In other words, the vacuum metric $(M=0)$ has the form of an AdS black hole such that in the context of AdS/CFT, it can be interpreted in terms of an entangled state of two copies of the CFT on a hyperbolic plane [91]. The entangled state of two copies of the CFT on a hyperbolic plane appearing above can then be understood as a conformally transformed description of the global vacuum state which entangles the CFT degrees of freedom on the interior with those on the exterior of the sphere.
This result provides strong motivation to investigate the vanishing of the complexity growth rate at some finite temperature other than zero. For $\beta=\beta_{\max }$, this is a minimum temperature given by $T_{\min }=r_{h} / 2 \pi$. Therefore, from Eq. (2.52) we expect that the variation of the complexity becomes zero at this temperature only in the Lloyd limit. There is a similar discussion on the temperature for the resistivity and conductivity of field theories which are dual to EMA-dilaton theories in Ref. [92].

We also note that regardless of the unphysical values obtained for the mass, in the case of $T<T_{\min }$ or $\beta>\beta_{\max }$, we observe that the equation $f(r)=0$ has two real positive roots and the geometry has a causal structure similar to that encountered for the charged black holes. We have plotted the behavior of the blackening factor $f(r)$ in four dimensions for different values of $\beta$ in Fig. 3. The case that happens here is denoted by the solid red curve in the figure. Studying the complexity growth rate for this chargedlike geometry similarly to what was done in the previous subsection, we find that $\dot{\mathcal{C}}_{A}$ vanishes at late times. It has been shown in Ref. [39] that a similar thing happens in the case of AdS black holes with hyperbolic geometry. For temperatures below $T=1 /(2 \pi L)$, the small hyperbolic black holes (i.e., $r_{h}<L$ ) have a causal structure similar to that of charged AdS black holes, and the late-time limit of $\dot{\mathcal{C}}_{A}$ goes to zero. In fact, though we consider the AdS branes with planar geometry in Eq. (2.3), for $\beta=\beta_{\max }$ the neutral brane metric behaves as well as a hyperbolic geometry (see the Appendix).

To analyze the behavior of the time evolution of the holographic complexity with more detail, we investigate the time derivative of the action in Eq. (2.50) as a function of time in the four-dimensional spacetime. The results are depicted for some typical locations of the event horizon, $r_{h}=1,1.5,2.5$, in Fig. 4(a) and for some values of the

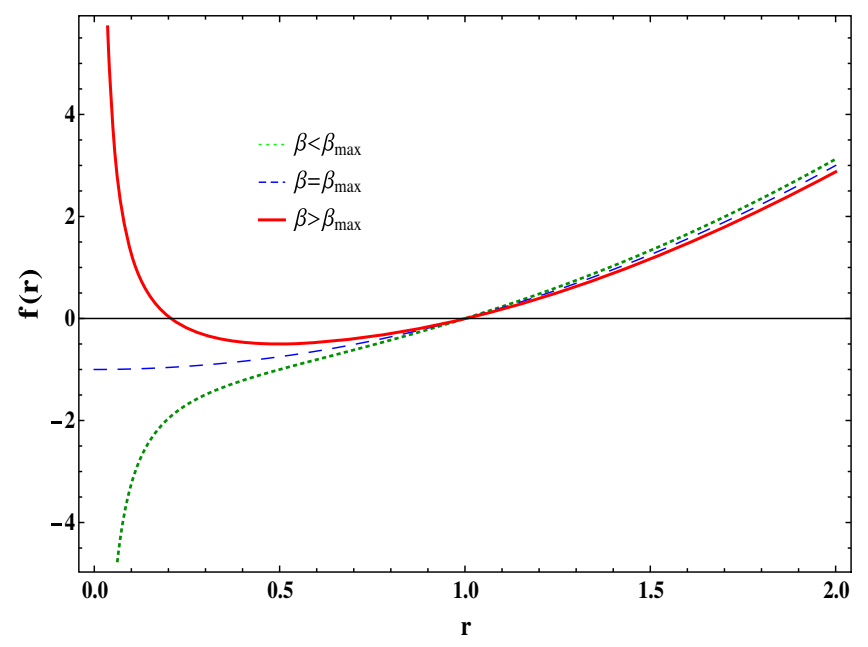

FIG. 3. The structure of the blackening factor for different values of $\beta$ with $d=3$ and $r_{h}=1$. The solid red line corresponds to $\beta>\beta_{\max }$. 


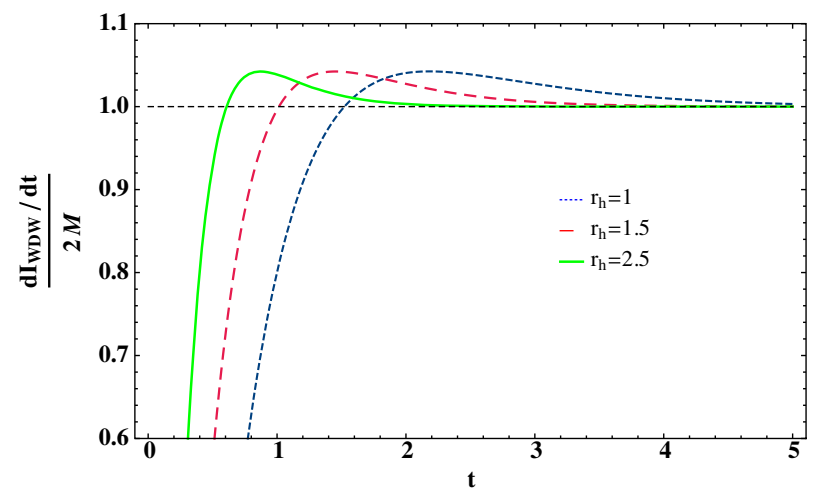

(a)

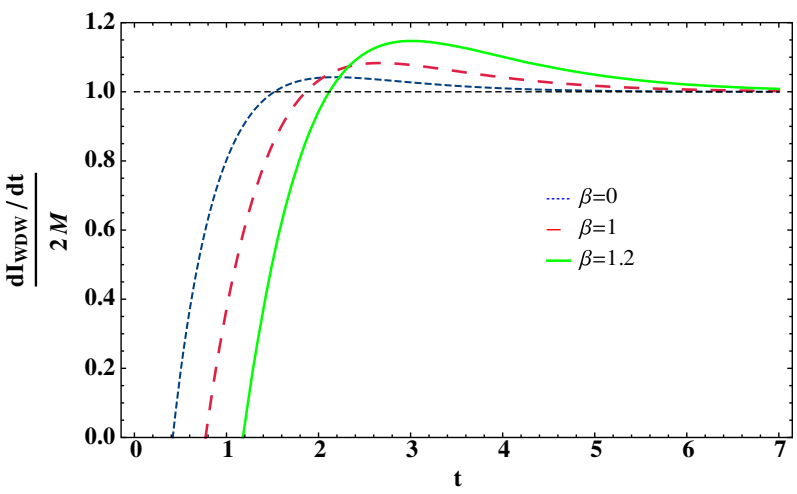

(b)

FIG. 4. The action growth rate on the WDW patch vs $t$ for different values of (a) $r_{h}$, and (b) $\beta$.

momentum relaxation constant, $\beta=0,1,1.2$, in Fig. 4(b). For the sake of numerical precision, we first solve the equation of $r_{m}$ in Eq. (2.51) numerically and then plot the diagrams. We can see from Fig. 4 that as the time passes from $t_{c}$, the growth rate of the action violates the Lloyd bound for all values of $r_{h}$ and $\beta$, while at large times $t \gg t_{c}$ it saturates the bound, i.e., $\dot{\mathcal{C}}_{A}=2 M$. Although the general behavior of the plots is the same, the curves behave differently in each panel. For instance, in the left panel, larger black holes saturate the bound sooner than smaller ones, while all violate the bound with the same strength (the curves have the same peak) in the initial times. In the right panel, the larger values of $\beta$ correspond to stronger violations of the bound, and they saturate the bound at somewhat later times. The other point that can be inferred from Figs. 4(a) and 4(b) is that the rate of complexity saturates the Lloyd bound from above at late times. It seems to be consistent with the positive sign of the second term in Eq. (2.50).

\section{Thermal diffusivity of neutral branes at minimal temperature}

From the holographic point of view in Ref. [15], black holes are regarded as the fastest computers in the sense that they saturate the complexification Lloyd bound [Eq. (1.4)]. In this regard, the scrambling time is a measure of how long it takes for information to spread through the system of $N$ degrees of freedom [77,93]. The rate of scrambling in a chaotic system is determined by a Lyapunov time, $\tau_{L} \sim$ $\hbar /\left(2 \pi k_{B} T\right)$ [77]. Quantum mechanics puts a bound on this exponent, and it has been shown in Refs. $[78,79,94]$ that the black holes saturate this bound. However, a fast computer should interact strongly, so a good candidate is a strongly coupled CFT in the context of the AdS/CMT. A class of such strongly coupled theories in quantum CM physics is the notion of strange metals with specific transport properties [95]. Now, the question is whether the chaos properties of black holes and many-body systems are connected to the transport coefficient.
It was proposed in Refs. [76,96] that one can reformulate the Kovtun-Son-Starinet bound (the bound on the ratio of shear viscosity to entropy density) [97] in terms of the diffusion constant as $\mathcal{D} \sim v_{B}^{2} \tau_{L}$, where $v_{B}$ is a characteristic velocity of the theory known as the butterfly velocity. For any holographic theory with a classical gravity dual, both the Lyapunov time and the butterfly velocity can be extracted from the properties of a black hole horizon [78,79]. On the other hand, thermal diffusivity provides a natural candidate to relate to many-body chaos, such that the relationship

$$
\mathcal{D}_{T} \geq v_{B}^{2} \tau_{L}
$$

is a generic low-temperature property of the homogeneous holographic lattice models [98]. Indeed, it is a universal piece of the diffusivity matrix that we can generically relate to the chaos exponents at infrared fixed points. It is defined as follows:

$$
\mathcal{D}_{T} \equiv \frac{\kappa}{c_{\rho}},
$$

where $\kappa$ is the open circuit thermal conductivity and $c_{\rho}$ is the thermodynamic specific heat at fixed density $\rho$. Though the thermal diffusion for neutral black holes in the fourdimensional EMA model with momentum relaxation has been recently studied slightly in Ref. [99], we consider this concept in general dimensions. On the other hand, we find that there is a minimum temperature for which the complexity growth rate vanishes, just like what happens in the case of AdS black holes with hyperbolic geometry in Ref. [39]. Therefore, it would be of interest to investigate the behavior of the transport parameters for these models at $T=T_{\min }$.

In the momentum relaxation model, $T_{\min }$ corresponds to $\beta_{\max }$, for which the mass of the black hole becomes zero. Thus, we can recast the growth rate of the holographic complexity in Eq. (2.52) as

$$
\dot{\mathcal{C}}_{A}=2 M=\frac{2(d-1)}{d-2} S\left(T-T_{\text {min }}\right),
$$


where $S$ and $T$ are the entropy and the temperature of neutral AdS black branes given in Eq. (2.38). Now, following Ref. [98], we can calculate the thermal conductivity and the specific heat, respectively, from

$$
\kappa=\left.4 \pi \frac{f^{\prime}(r) r^{2(d-2)}}{\left(f^{\prime}(r) r^{d-2}\right)^{\prime}}\right|_{r=r_{h}} \quad \text { and } \quad c_{\rho}=T \frac{\partial s}{\partial T},
$$

where $s=S / V_{d-1}$ is the entropy density and we set $16 \pi G=1$ in the rest of this section and the Appendix. Substituting the blackening factor [Eq. (2.3)] with $q=0$ and Eq. (2.38) into Eq. (2.56) then yields

$$
\begin{aligned}
\kappa & =4 \pi r_{h}^{d-2} \frac{\left(-2 d r_{h}^{2}+\beta^{2}\right)}{2 d(d-3) r_{h}^{2}-(d-1) \beta^{2}}, \\
c_{\rho} & =4 \pi(d-1) r_{h}^{d-1} \frac{\left(2 d r_{h}^{2}-\beta^{2}\right)}{\left(2 d r_{h}^{2}+\beta^{2}\right)} .
\end{aligned}
$$

Now, if one inserts these parameters into Eq. (2.54), the diffusion constant is given by

$$
\mathcal{D}_{T}=\frac{1}{(d-1) r_{h}} \frac{\left(2 d r_{h}^{2}+\beta^{2}\right)}{\left((d-1) \beta^{2}-2 d(d-3) r_{h}^{2}\right)} .
$$

For the minimum value of the temperature where $\beta_{\max }^{2}=2(d-2) r_{h}^{2}$, the above transport parameters are reduced to

$$
\kappa=4 \pi r_{h}^{d-2}, \quad c_{\rho}=4 \pi r_{h}^{d-1}, \quad \mathcal{D}_{T}=\frac{1}{r_{h}} .
$$

The calculations for the AdS hyperbolic black holes are left to the Appendix. From the relationsin Eqs. (2.59) and (A8), one can find that irrespective of the dimension of spacetime, we have the same expressions for these quantities in both solutions at the minimum temperature. In particular, the diffusion constants are independent of the spacetime dimensions and are proportional to the inverse of the horizon radius. Such an effect has long been similarly established in Ref. [100] — that the dc transport coefficients of conserved quantities is related to the horizon via the membrane paradigm.

As previously mentioned, there is a deep connection between the transport coefficients and the chaos parameters of any holographic geometry. Moreover, these parameters can be extracted by analyzing a shockwave propagating on the black hole horizon $[78,79,96]$. In units $\hbar=k_{B}=1$, the Lyapunov time is given by $\tau_{L} \sim(2 \pi T)^{-1}$, and the butterfly velocity can be calculated in the near horizon of the isotropic metric [Eq. (2.2)] as [101,102]

$$
v_{B}^{2}=\left.\frac{f(r)^{\prime}}{2(d-1) r}\right|_{r_{h}}=\frac{2 d r_{h}^{2}-\beta^{2}}{4(d-1) r_{h}^{2}},
$$

where we have used the blackening factor [Eq. (2.3)] with $q=0$ for neutral black branes. At the minimum temperature, or equivalently $\beta_{\max }^{2}=2(d-2) r_{h}^{2}$, this velocity becomes

$$
v_{B}=\sqrt{\frac{1}{d-1}}=\sqrt{\frac{2}{d}} v_{B}^{\text {Sch }},
$$

where $v_{B}^{\text {Sch }}$ is the value of the butterfly velocity for an AdSSchwarzschild black brane in $d+1$ dimensions [78]. As shown, the velocity in EMA theory at this special point is lower than its counterpart in Einstein gravity for $d \geq 3$. The diffusion constant in Eq. (2.59), together with parameters $v_{B}$ and $\tau_{L}$ at the minimal temperature $T_{\min }=r_{h} / 2 \pi$, respects the bound in Eq. (2.53)-i.e.,

$$
\frac{D_{T}}{\tau_{L} v_{B}^{2}}=(d-1) \geq 1 \text {. }
$$

As is obvious, they saturate the bound only for $d=2$ in three-dimensional spacetime.

\section{THE GROWTH RATE OF COMPLEXITY IN THE $k$-ESSENCE SECTOR}

The previous studies can be generalized to the case in which the kinetic term for the scalar fields can have nonlinear contributions. Such a case can be implemented by the so-called $k$-essence models [80], in which the mentioned kinetic term is generalized to be a function $P\left(\psi,(\partial \psi)^{2}\right)$. A simple case contained in this setup is that the scalar fields apart from the standard kinetic term possess a kinetic nonlinear contribution given by the higher powers of the kinetic term. In this section, we are going to study the growth rate of complexity for the dyonic $\operatorname{AdS}$ black branes in this holographic model from the CA proposal. However, for later convenience we will consider the four-dimensional bulk spacetime.

The action of the nonlinear EMA theory was studied in Refs. [81,82] and is given by

$$
\begin{aligned}
I_{\mathrm{bulk}}= & \frac{1}{16 \pi G} \int_{M} d^{4} x \sqrt{-g}\left[R-2 \Lambda-\frac{1}{4} F_{\mu \nu} F^{\mu \nu}\right. \\
& \left.-\sum_{I=1}^{2}\left(\chi_{I}+\gamma \chi_{I}^{k}\right)\right]
\end{aligned}
$$

where $\chi_{I}=\frac{1}{2} \partial_{\mu} \psi_{I} \partial^{\mu} \psi_{I}$, and $\gamma$ is the coupling of the nonlinear axionic term. The AdS black brane solution of this model is described by the ansatz in Eq. (2.2). Therefore, the blackening factor and the axionic scalar fields are

$$
\begin{aligned}
f(r) & =r^{2}-\frac{\beta^{2}}{2}+\frac{Q_{e}^{2}+Q_{m}^{2}}{4 r^{2}}-\frac{2 m_{0}}{r}+\gamma \frac{\beta^{2 k}}{2^{k}(2 k-3) r^{2(k-1)}}, \\
\psi_{1} & =\beta x_{1}, \quad \psi_{2}=\beta x_{2} .
\end{aligned}
$$


The Maxwell equation is easily solved by

$$
A=-\frac{Q_{e}}{r} d t+\frac{Q_{m}}{2}\left(x_{1} d x_{2}-x_{2} d x_{1}\right),
$$

where $Q_{e}$ and $Q_{m}$ are the electric and magnetic monopole charges. The temperature and the entropy of the solution are given by

$$
\begin{aligned}
T & =\frac{f^{\prime}\left(r_{+}\right)}{4 \pi}=\frac{1}{4 \pi}\left(3 r_{+}-\frac{\beta^{2}}{2 r_{+}}-\frac{Q_{e}^{2}+Q_{m}^{2}}{4 r_{+}^{3}}-\gamma \frac{\beta^{2 k}}{2^{k} r_{+}^{2 k-1}}\right), \\
S & =\frac{V_{2}}{16 \pi G} 4 \pi r_{+}^{2} .
\end{aligned}
$$

Also, the mass parameter is obtained from the condition $f\left(r_{+}\right)=0$, such that the mass of the solution becomes

$$
\begin{aligned}
M & =\frac{4 V_{2}}{16 \pi G} m_{0}, \\
m_{0} & =\frac{Q_{e}^{2}+Q_{m}^{2}}{8 r_{+}}+\frac{1}{2} r_{+}^{3}-\frac{1}{4} r_{+} \beta^{2}-\gamma \frac{\beta^{2 k}}{2^{k+1}(2 k-3) r_{+}^{2 k-3}},
\end{aligned}
$$

and $r_{+}$is the location of the event horizon. The extended thermodynamics of the above solution has been studied in Refs. [82,103]. We note also that in particular, for $k=2$ the metric [Eq. (3.2)] behaves as a double-horizon black hole (i.e., $r_{+}$and $r_{-}$), so one can use the WDW patch in Fig. 1 to compute the evolution of the holographic complexity of state which is dual to this geometry.

\section{A. The action growth rate}

The total time derivative of the holographic complexity for dyonic charged AdS solutions in this model is calculated from Eq. (2.33). However, as mentioned in the previous section, we can also consider the contribution of a boundary term for the Maxwell field in the total action as [90]

$$
I_{\mu Q}=\eta \int_{\partial \mathcal{M}} d \Sigma_{\mu} F^{\mu \nu} A_{\nu},
$$

which does not change the equations of motion. In general, employing a Dirichlet boundary condition results in a wellposed variational principle, but due to the boundary term (3.6) we instead need to impose a Neumann boundary condition for $\eta=1$, or a mixed boundary condition for general $\eta$. A comprehensive discussion about this boundary action is given in Ref. [90]. On the other hand, using the Stokes theorem and the Maxwell equations, we can convert the boundary term in Eq. (3.6) to the bulk Maxwell action as

$$
I_{\mu Q}=\frac{\eta}{2} \int_{\mathcal{M}} d^{4} x \sqrt{-g} F_{\mu \nu} F^{\mu \nu} .
$$

Therefore, the boundary action (3.6) contributes in the complexity growth rate through this bulk term.
Using similar discussion for the three bulk regions on the WDW patch in Fig. 1, the complexity of the bulk action is written as follows:

$$
\begin{aligned}
I_{\mathrm{bulk}}+I_{\mu Q}= & \frac{V_{2}}{16 \pi G} \int_{\mathrm{WDW}} d r d t r^{2}\left[-6-\gamma \frac{\beta^{4}}{2 r^{4}}\right. \\
& \left.-(2 \eta-1) \frac{Q_{e}^{2}-Q_{m}^{2}}{2 r^{4}}\right],
\end{aligned}
$$

where in four dimensions the volume of the boundary surface becomes $V_{2}$, then for the time evolution of the bulk term we have

$$
\frac{d}{d t}\left(\delta I_{\mathrm{Bulk}}+I_{\mu Q}\right)=\left.\frac{V_{2}}{8 \pi G}\left[r^{3}-\gamma \frac{\beta^{4}}{4 r}-(2 \eta-1) \frac{Q_{e}^{2}-Q_{m}^{2}}{4 r}\right]\right|_{r_{m}^{2}} ^{r_{m}^{1}} .
$$

For the joints of null boundaries in $r_{m}^{1}$ and $r_{m}^{2}$, we have

$$
\frac{d I_{\text {joint }}}{d t}=-\left.\frac{V_{2}}{8 \pi G}\left[r^{3}-\frac{Q_{e}^{2}+Q_{m}^{2}}{4 r}-\gamma \frac{\beta^{4}}{4 r}+r f(r) \log \frac{|f(r)|}{\xi^{2}}\right]\right|_{r_{m}^{2}} ^{r_{m}^{1}},
$$

and also the contribution of the counterterm action becomes

$$
\frac{d I_{\mathrm{ct}}}{d t}=\left.\frac{V_{2}}{8 \pi G}\left[4 m_{0}-\frac{Q_{e}^{2}+Q_{m}^{2}}{2 r}-\frac{\beta^{4} \gamma}{2 r}+\beta^{2} r-2 r^{3}\right] \log \frac{2 \ell_{c} \xi}{r}\right|_{r_{m}^{2}} ^{r_{m}^{1}} .
$$

As asserted in the previous section, the contribution of the GHY boundary terms for cutoff surfaces on the WDW patch in Fig. 1 is time independent; thus, we ignore them. By combining the above results, we can determine the final expression for the complexity growth rate as

$$
\frac{d \mathcal{C}_{A}}{d t}=\frac{V_{2}}{8 \pi G}\left[\frac{(1-\eta) Q_{e}^{2}+\eta Q_{m}^{2}}{2 r}-r f(r) \log \frac{4 \ell_{c}^{2}|f(r)|}{r^{2}}\right]_{r_{m}^{2}}^{r_{m}^{1}} .
$$

It is obvious that the normalization constant $\xi$ and the coupling of the nonlinear axionic action $\gamma$ do not affect the final result explicitly. Also, one can check that in the limit $\gamma \rightarrow 0$, Eqs. (3.9)-(3.11) give the results in the previous subsection for the electrically $\left(Q_{m}=0\right)$ charged $\operatorname{AdS}_{d+1}$ black branes when $d=3$. According to Eq. (3.9), for $\eta=1 / 2$, the boundary and the bulk Maxwell terms cancel each other and do not affect the gravitational action, in spite of their geometric contribution in the background [Eq. (3.2)] to the action. 


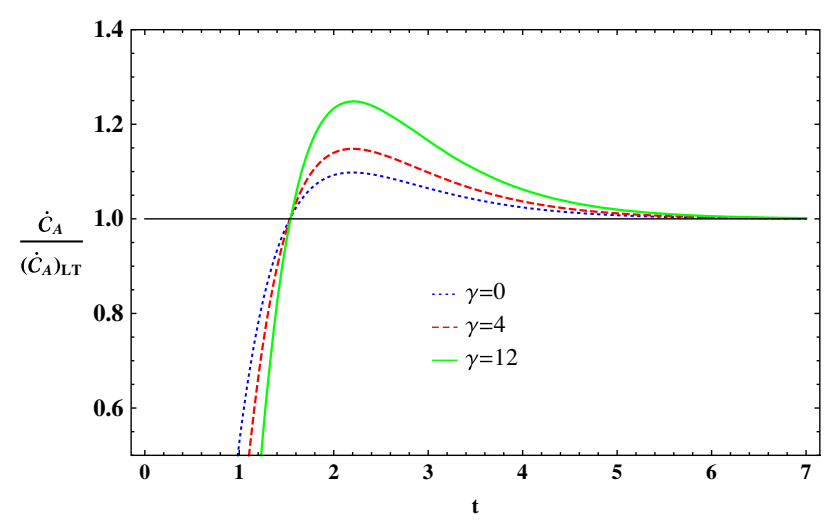

(a) $Q_{e} / Q_{m}=2$

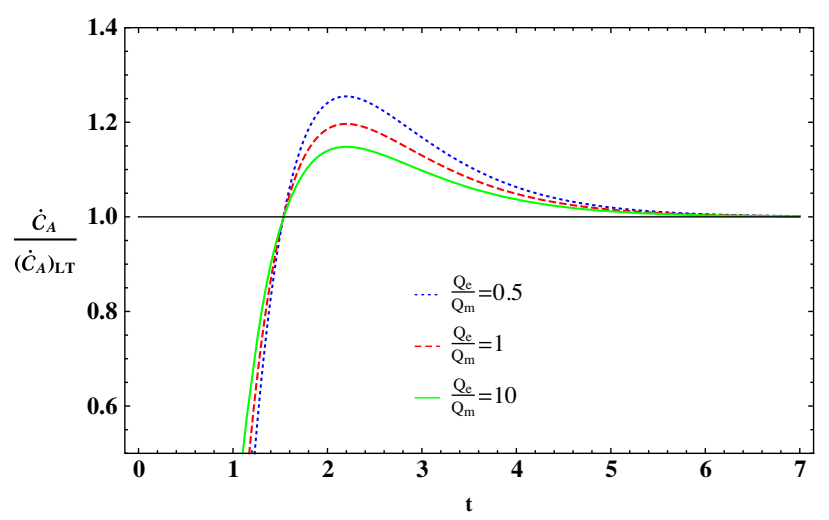

(b) $\gamma=4$

FIG. 5. Lloyd bound violation for different values of $\gamma$ and $Q_{e} / Q_{m}$ with $\beta=0.2, \eta=0.2, \ell_{c}=1$.

\section{B. The late-time behavior}

Since in the late-time limit the meeting points reach to the horizons of the geometry on the WDW patch, as shown in Fig. 1, the final result for the growth rate of complexity is

$$
\left.\frac{d \mathcal{C}_{A}}{d t}\right|_{\gg \gg t_{c}}=\frac{V_{2}}{16 \pi G}\left[\frac{(1-\eta) Q_{e}^{2}+\eta Q_{m}^{2}}{r}\right]_{r_{+}}^{r_{-}} .
$$

The result shows that in the absence of the Maxwell boundary term (i.e., $\eta=0$ ), the late-time behavior is similar to the case of charged black branes in Eq. (2.35) even in the presence of the magnetic charge. Also for $\eta=1$, the late-time growth rate is only proportional to the magnetic charge-i.e., it vanishes for electrically charged black branes.

To better understand the full time dependence of Eq. (3.12), it is straightforward to provide a numerical study on the growth rate of complexity for the dyonic black holes described by. Eq. (3.2). In this respect, we have plotted the ratio $\dot{\mathcal{C}}_{A} /\left(\dot{\mathcal{C}}_{A}\right)_{L T}$ for different values of $\gamma$, $Q_{e} / Q_{m}$, and $\eta$ in Figs. 5 and 6. Here, $L T$ stands for the

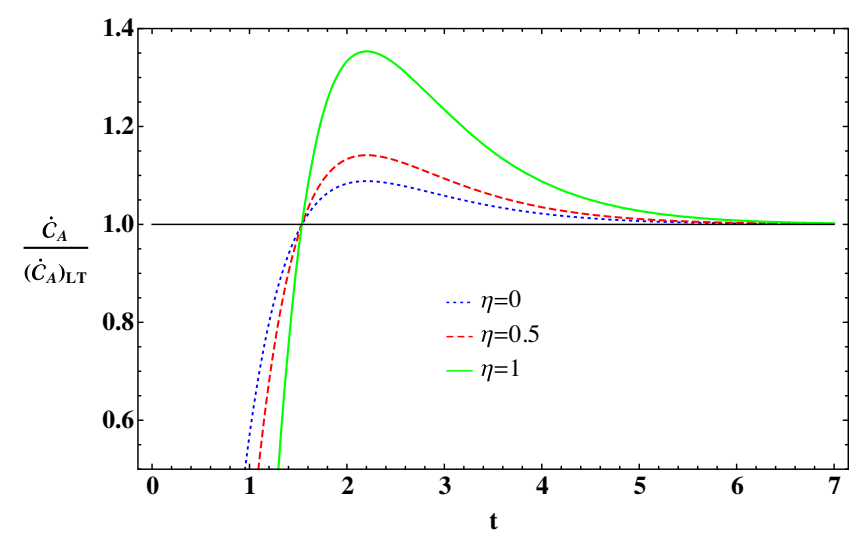

FIG. 6. Lloyd bound violation for different values of $\eta$ with $Q_{e} / Q_{m}=2, \beta=0.2, \gamma=5, \ell_{c}=1$. late-time behavior, and $\left(\dot{\mathcal{C}}_{A}\right)_{L T}$ is given in Eq. (3.13). The figures show that the bound is violated due to the fact that $\dot{\mathcal{C}}_{A}$ approaches the bound at very late times from above.

We see from Fig. 5(a) that irrespective of the behavior of $\dot{\mathcal{C}}_{A}$ at the initial times, the larger the value of the nonlinear axionic term, the stronger the violation of the bound. In contrast, by increasing the ratio between the electric and the magnetic charges, the violation becomes weaker, as shown in Fig. 5(b). This opposite behavior relative to these constant parameters is expected due to the relations (3.2) and (3.12). In other words, for a dominant electrical solution (i.e., $\eta<1 / 2$ ), the signs of the charge ratio and $\gamma$ term are different. Of course, we have checked that this opposition also occurs for a dominant magnetic solution $(\eta>1 / 2)$. We have checked that the behavior of $\dot{\mathcal{C}}_{A} /\left(\dot{\mathcal{C}}_{A}\right)_{L T}$ for charged black branes, obtained in Eq. (2.34) via CA conjecture, is very similar to the dotted plot shown in Fig. 5(a) with $\gamma=0$ and $Q_{m}=0$. In Fig. 6, we illustrate the effect of the boundary Maxwell action [Eq. (3.6)] with coupling $\eta$. As observed, when one increases the value of $\eta$, the Lloyd bound for these charged solutions is violated from above drastically, just like what happens for the values of the nonlinear axionic term in Fig. 5(a).

\section{CONCLUSIONS AND OUTLOOK}

In this paper, we extended the study of holographic complexity via AdS black branes with momentum relaxation into $(d+1)$-dimensional EMA theory by using the $\mathrm{CA}$ conjecture. The momentum relaxation introduced by linear massless axion fields in the bulk breaks the translational symmetry of the dual field theory and gives finite conductivity. In this regard, to retain the homogeneity of the bulk theory, the axion fields have been assumed to be a linear function of the boundary spatial coordinates, with the equal constant coefficients determining the strength of the momentum relaxation. Particularly, we have investigated the effects of this parameter on the holographic complexity and its time evolution. 
We computed the complexity for both the charged and neutral branes as homogeneous and isotropic solutions of the bulk theory. Following the approach in Ref. [23] to evaluate the complexity on the WDW patch in each sector, we considered the general GHY surface terms and the contribution of joints and counterterms of the corresponding null boundaries. The late-time behavior of the growth rate of the holographic complexity was studied for two solutions, and we found that it is always finite and well defined, and satisfies the Lloyd bound in Eqs. (2.35) and (2.52). It has been found in Ref. [14] that the action growth rate at late times for neutral AdS black holes is $d I_{\mathrm{WDW}} / d t=2 M$, independent of the size of the black hole and the spacetime dimension. We observed that the neutral AdS black brane confirms this statement even in the presence of momentum relaxation.

We also obtained an upper bound for the strength of the momentum relaxation $\beta_{\max }$, in the case of neutral branes, such that for $\beta>\beta_{\max }$ the brane mass becomes negative, which has no physical meaning. We studied the time evolution of the complexity numerically in Fig. 3 for different values of $r_{h}$ and $\beta$. The plots showed that in spite of different rates, the complexity growth rates for different sizes of the brane and different strengths of the momentum relaxation saturate the Lloyd bound from above. An outstanding outcome corresponding to $\beta_{\max }$ is that there is a minimum temperature given by $T_{\min }=r_{h} / 2 \pi$, for which the growth rate of complexity vanishes, in spite of the fact that it should vanish at zero temperature for neutral AdS branes. In a separate development, remarkable connections have been pointed out between the dynamics of black holes and the nature of quantum chaos in many-body quantum systems in the context of holographic correspondence. We computed the thermal conductivity and diffusivity for neutral AdS black branes and hyperbolic black holes in general $d+1$ dimensions as well. We have shown that at the minimum temperature, the diffusion constants are proportional to the inverse of the event horizon radius, independent of the dimension of spacetime. Also, this coefficient accompanied with the chaotic parameters respected the corresponding bound in the $\mathrm{CM}$ physics and saturated this bound at minimum temperature only in the case of three dimensions.

In addition, we have studied a holographic model including the nonlinear contribution of an axionic kinetic term while preserving the homogeneity and isotropy of the solutions. We assumed a particular branch of solutions that has been described by a dyonic charged black brane with momentum relaxation, and then calculated the rate of complexity in this model. We have also considered a boundary action for the Maxwell field in this model. The Maxwell boundary term contributed as a bulk action in the change of complexity, such that for $\eta=1 / 2$, the bulk actions had no contribution in the complexity rate. The results showed that even though the coupling of the nonlinear term $(\gamma)$ affects the contribution of different actions in the total complexity, it does not change the growth rate at late times as denoted in Eq. (3.13).

In the absence of a Maxwell surface term $(\eta=0)$, the growth rate vanished at late time for purely magnetic charged branes, while for nonzero electric charges it gave the known expression for general charged branes as in Eq. (2.35). In contrast, for $\eta=1$ the behavior is reversedthat is, the rate of growth is nonvanishing for pure magnetically charged branes and vanishing for electrically charged ones. The numerical investigation for the full time dependence of the complexity in the CA proposal is illustrated in Figs. 5 and 6 . The results show that the Lloyd bound is violated due to the fact that $\dot{\mathcal{C}}_{A}$ approaches the bound at very late times from above, even for different values of $\gamma, Q_{e} / Q_{m}$, and $\eta$, of course with different rates.

It would be of interest to consider the effects of momentum relaxation on the growth rate of complexity in the case of charged dilatonic backgrounds. In general, the action (2.1) in the presence of dilaton and axion fields with momentum relaxation recasts as follows [92]:

$$
\begin{aligned}
I= & \frac{1}{16 \pi G} \int_{M} d^{d+1} x \sqrt{-g}\left[R-\frac{1}{2}(\partial \phi)^{2}+V(\phi)\right. \\
& \left.-\frac{1}{4} Z(\phi) F_{\mu \nu} F^{\mu \nu}-\frac{1}{2} \sum_{j}^{d-1}\left(\partial \psi_{j}\right)^{2}\right] .
\end{aligned}
$$

Related discussions about the growth of the holographic complexity for dilatonic metrics without axion fields have been done in Ref. [25]. For instance, it has been shown in Ref. [50] that the total rate of the holographic complexity at late times is given by

$$
\left.\frac{d \mathcal{C}_{A}}{d t}\right|_{t \rightarrow \infty}=2 M-\mu Q-D, \quad D \equiv \frac{e^{2 \phi} Q^{2}}{2 M} .
$$

We suggest that adding the axionic action, as in Eq. (4.1), will change the structure of the Lloyd bound of complexity given in Eq. (4.2) by a term like $D$; however, we postpone the study of this proposal for future works.

One can also study the complexity growth rate of a nonrelativistic but isotropic boundary theory [104] which is dual to a bulk geometry with momentum relaxation for the Lifshitz and hyperscaling-violating metrics, as done in Ref. [105] for Einstein-Maxwell-Dilaton theory without momentum relaxation. Another proposal in the context of CA conjecture for these holographic models is to investigate the complexity growth rate of AdS black branes at a finite cutoff. The concept of this geometric cutoff at $r=r_{c}$ comes from the $T \bar{T}$ deformation of a CFT in the AdS/CFT dictionary [106,107], such that the coupling of this operator removes the asymptotic region of the AdS spacetime. In this regard, some efforts have been made in Refs. [108-110]. 


\section{ACKNOWLEDGMENTS}

The authors would like to thank M. Alishahiha, A. Ghodsi, M. R. Mohammadi Mozaffar, and G. Jafari for valuable comments and discussions. H. B.-A. and H. M. would like to thank Z. Ebadi, M. Nattagh Najafi, and M. Maleki for useful discussions.

\section{APPENDIX: THERMAL DIFFUSIVITY OF HYPERBOLIC BLACK HOLES}

In this appendix, we consider the thermodynamics and transport properties of neutral AdS black holes in $d+1$ dimensions with hyperbolic geometry. Following the convention in Ref. [39], the metric with spherical symmetry takes the general form

$$
d s^{2}=-f(r) d t^{2}+\frac{d r^{2}}{f(r)}+r^{2} d \Sigma_{k, d-1}^{2},
$$

where the blackening factor is given by

$$
f(r)=\frac{r^{2}}{L^{2}}+k-\frac{\omega^{d-2}}{r^{d-2}},
$$

in which $\omega$ is the mass parameter and $L$ denotes the AdS curvature scale. $d \Sigma_{k, d-1}^{2}$ is the $(d-1)$-dimensional line element of curvature $k=\{+1,0,-1\}$ so that the black holes corresponding to $k=\{+1,0,-1\}$ have spherical, planar, and hyperbolic horizons, respectively.

Here, we are interested in the case $k=-1$, where $d \Sigma_{-1, d-1}^{2}=d \theta^{2}+\sinh ^{2} \theta d \Omega_{d-2}^{2}$ is the metric on a $(d-1)$-dimensional hyperbolic plane. The mass of the black hole is given by

$$
M=\frac{(d-1) V_{d-1}}{16 \pi G} r_{h}^{d-2}\left(\frac{r_{h}^{2}}{L^{2}}-1\right),
$$

where $V_{d-1}$ denotes the dimensionless volume of the relevant spatial geometry and $r_{h}$ is the event horizon of the black hole whose position is the largest root of $f\left(r_{h}\right)=0$.

The entropy and Hawking temperature of the black hole are

$$
\begin{aligned}
& S=\frac{A_{h}}{4 G}=\frac{V_{d-1}}{4 G} r_{h}^{d-1}, \\
& T=\left.\frac{f^{\prime}(r)}{4 \pi}\right|_{r=r_{h}}=\frac{1}{4 \pi r_{h}}\left(d \frac{r_{h}^{2}}{L^{2}}-(d-2)\right) .
\end{aligned}
$$

The minimum temperature for which the rate of growing the holographic complexity vanishes is given by $T_{\min }=$ $\frac{1}{2 \pi L}$. Therefore, one can rewrite its rate at the late-time limit as

$$
\dot{\mathcal{C}}_{A}=2 M=\frac{2(d-1)}{d} S\left(T-T_{\text {min }}\right)
$$

Now, we consider the thermal conductivity and diffusivity for hyperbolic black holes at this minimal temperature. From the definitions in Eq. (2.56) and the entropy and temperature in Eq. (A4), we have

$$
\begin{aligned}
\kappa & =4 \pi r_{h}^{d-2} \frac{\left(d r_{h}^{2}-(d-2) L^{2}\right)}{d(d-3) r_{h}^{2}-(d-1)(d-2) L^{2}}, \\
c_{\rho} & =4 \pi(d-1) r_{h}^{d-1} \frac{\left(d r_{h}^{2}-(d-2) L^{2}\right)}{\left(d r_{h}^{2}+(d-2) L^{2}\right)} .
\end{aligned}
$$

Also, Eq. (2.54) yields the diffusion constant

$\mathcal{D}_{T}=-\frac{1}{(d-1) r_{h}} \frac{d r_{h}^{2}+(d-2) L^{2}}{\left(d(d-3) r_{h}^{2}-(d-1)(d-2) L^{2}\right)}$.

Finally, we obtain the following expressions in the minimum temperature for which $L=r_{h}$ :

$$
\kappa=4 \pi r_{h}^{d-2}, \quad c_{\rho}=4 \pi r_{h}^{d-1}, \quad \mathcal{D}_{T}=\frac{1}{r_{h}} .
$$

[1] J. M. Maldacena, The large- $N$ limit of superconformal field theories and supergravity, Int. J. Theor. Phys. 38, 1113 (1999); Adv. Theor. Math. Phys. 2, 231 (1998).

[2] E. Witten, Anti-de Sitter space and holography, Adv. Theor. Math. Phys. 2, 253 (1998).

[3] S. S. Gubser, I. R. Klebanov, and A. M. Polyakov, Gauge theory correlators from noncritical string theory, Phys. Lett. B 428, 105 (1998).

[4] G. 't Hooft, Dimensional reduction in quantum gravity, Conf. Proc. C 930308, 284 (1993) [arXiv:gr-qc/9310026].
[5] L. Susskind, The world as a hologram, J. Math. Phys. (N.Y.) 36, 6377 (1995).

[6] S. Ryu and T. Takayanagi, Holographic Derivation of Entanglement Entropy from AdS/CFT, Phys. Rev. Lett. 96, 181602 (2006).

[7] S. Ryu and T. Takayanagi, Aspects of holographic entanglement entropy, J. High Energy Phys. 08 (2006) 045.

[8] T. Faulkner, M. Guica, T. Hartman, R. C. Myers, and M. Van Raamsdonk, Gravitation from entanglement in holographic CFTs, J. High Energy Phys. 03 (2014) 051. 
[9] L. Susskind, Entanglement is not enough, Fortsch. Phys. 64, 49 (2016).

[10] J. Watrous, in Quantum Computational Complexity, Encyclopedia of Complexity and Systems Science, edited by R. A. Meyers (Springer, New York, 2009), pp. 7174-7201.

[11] S. Aaronson, The complexity of quantum states and transformations: From quantum money to black holes, arXiv:1607.05256.

[12] L. Susskind, Computational complexity and black hole horizons, Fortschr. Phys. 64, 24 (2016).

[13] D. Stanford and L. Susskind, Complexity and shock wave geometries, Phys. Rev. D 90, 126007 (2014).

[14] A. R. Brown, D. A. Roberts, L. Susskind, B. Swingle, and Y. Zhao, Holographic Complexity Equals Bulk Action?, Phys. Rev. Lett. 116, 191301 (2016).

[15] A. R. Brown, D. A. Roberts, L. Susskind, B. Swingle, and Y. Zhao, Complexity, action, and black holes, Phys. Rev. D 93, 086006 (2016).

[16] G. Hayward, Gravitational action for space-times with nonsmooth boundaries, Phys. Rev. D 47, 3275 (1993).

[17] L. Lehner, R. C. Myers, E. Poisson, and R. D. Sorkin, Gravitational action with null boundaries, Phys. Rev. D 94, 084046 (2016).

[18] J. M. Maldacena, Eternal black holes in anti-de Sitter, J. High Energy Phys. 04 (2003) 021.

[19] J. Maldacena and L. Susskind, Cool horizons for entangled black holes, Fortsch. Phys. 61, 781 (2013).

[20] S. Lloyd, Ultimate physical limits to computation, Nature (London) 406, 1047 (2000).

[21] N. Margolus and L. B. Levitin, The maximum speed of dynamical evolution, Physica (Amsterdam) 120D, 188 (1998).

[22] R. G. Cai, S. M. Ruan, S. J. Wang, R. Q. Yang, and R. H. Peng, Action growth for AdS black holes, J. High Energy Phys. 09 (2016) 161.

[23] D. Carmi, S. Chapman, H. Marrochio, R. C. Myers, and S. Sugishita, On the time dependence of holographic complexity, J. High Energy Phys. 11 (2017) 188.

[24] R. Q. Yang, C. Niu, C. Y. Zhang, and K. Y. Kim, Comparison of holographic and field theoretic complexities for time dependent thermofield double states, J. High Energy Phys. 02 (2018) 082.

[25] B. Swingle and Y. Wang, Holographic complexity of Einstein-Maxwell-Dilaton gravity, J. High Energy Phys. 09 (2018) 106.

[26] Y.S. An and R. H. Peng, Effect of the dilaton on holographic complexity growth, Phys. Rev. D 97, 066022 (2018).

[27] S. P. Jordan, Fast quantum computation at arbitrarily low energy, Phys. Rev. A 95, 032305 (2017).

[28] W. Cottrell and M. Montero, Complexity is simple!, J. High Energy Phys. 02 (2018) 039.

[29] S. Deffner and S. Campbell, Quantum speed limits: From Heisenberg's uncertainty principle to optimal quantum control, J. Phys. A 50, 453001 (2017).

[30] A. R. Brown and L. Susskind, Second law of quantum complexity, Phys. Rev. D 97, 086015 (2018).

[31] J. Couch, S. Eccles, W. Fischler, and M. L. Xiao, Holographic complexity and noncommutative gauge theory, J. High Energy Phys. 03 (2018) 108.
[32] M. Moosa, Divergences in the rate of complexification, Phys. Rev. D 97, 106016 (2018).

[33] S. Mahapatra and P. Roy, On the time dependence of holographic complexity in a dynamical Einstein-dilaton model, J. High Energy Phys. 11 (2018) 138.

[34] Y. S. An, R. G. Cai, and Y. Peng, Time dependence of holographic complexity in Gauss-Bonnet gravity, Phys. Rev. D 98, 106013 (2018).

[35] M. Alishahiha, Holographic complexity, Phys. Rev. D 92 , 126009 (2015).

[36] D. Carmi, R. C. Myers, and P. Rath, Comments on holographic complexity, J. High Energy Phys. 03 (2017) 118.

[37] O. Ben-Ami and D. Carmi, On volumes of subregions in holography and complexity, J. High Energy Phys. 11 (2016) 129.

[38] M. Alishahiha, K. Babaei Velni, and M. R. Mohammadi Mozaffar, Black hole subregion action and complexity, Phys. Rev. D 99, 126016 (2019).

[39] S. Chapman, H. Marrochio, and R. C. Myers, Complexity of formation in holography, J. High Energy Phys. 01 (2017) 062.

[40] A. Reynolds and S. F. Ross, Divergences in holographic complexity, Classical Quantum Gravity 34, 105004 (2017).

[41] R. Q. Yang, C. Niu, and K. Y. Kim, Surface counterterms and regularized holographic complexity, J. High Energy Phys. 09 (2017) 042.

[42] M. Alishahiha, K. Babaei Velni, and M. R. Tanhayi, Complexity and near extremal charged black branes, Ann. Phys. (Amsterdam) 425, 168398 (2021).

[43] M. Alishahiha, A. Faraji Astaneh, A. Naseh, and M. H. Vahidinia, On complexity for $\mathrm{F}(\mathrm{R})$ and critical gravity, J. High Energy Phys. 05 (2017) 009.

[44] P. A. Cano, R. A. Hennigar, and H. Marrochio, Complexity Growth Rate in Lovelock Gravity, Phys. Rev. Lett. 121, 121602 (2018).

[45] J. Jiang, Action growth rate for a higher curvature gravitational theory, Phys. Rev. D 98, 086018 (2018).

[46] J. Jiang and H. Zhang, Surface term, corner term, and action growth in $F\left(R_{a b c d}\right)$ gravity theory, Phys. Rev. D 99, 086005 (2019).

[47] J. Jiang and B. Deng, Investigating the holographic complexity in Einsteinian cubic gravity, Eur. Phys. J. C 79, 832 (2019).

[48] A. Ghodsi, S. Qolibikloo, and S. Karimi, Holographic complexity in general quadratic curvature theory of gravity, Eur. Phys. J. C 80, 920 (2020).

[49] S. A. Hosseini Mansoori and M. M. Qaemmaqami, Complexity growth, butterfly velocity and black hole thermodynamics, Ann. Phys. (Amsterdam) 419, 168244 (2020).

[50] R. G. Cai, M. Sasaki, and S. J. Wang, Action growth of charged black holes with a single horizon, Phys. Rev. D 95, 124002 (2017).

[51] S. Chapman, M. P. Heller, H. Marrochio, and F. Pastawski, Toward a Definition of Complexity for Quantum Field Theory States, Phys. Rev. Lett. 120, 121602 (2018).

[52] R. Jefferson and R.C. Myers, Circuit complexity in quantum field theory, J. High Energy Phys. 10 (2017) 107.

[53] B. Czech, Einstein Equations from Varying Complexity, Phys. Rev. Lett. 120, 031601 (2018). 
[54] A. Belin, A. Lewkowycz, and G. Srosi, Complexity and the bulk volume, a New York time story, J. High Energy Phys. 03 (2019) 044.

[55] S. Chapman, J. Eisert, L. Hackl, M. P. Heller, R. Jefferson, H. Marrochio, and R. C. Myers, Complexity and entanglement for thermofield double states, SciPost Phys. 6, 034 (2019).

[56] R. Khan, C. Krishnan, and S. Sharma, Circuit complexity in fermionic field theory, Phys. Rev. D 98, 126001 (2018).

[57] H. A. Camargo, M. P. Heller, R. Jefferson, and J. Knaute, Path Integral Optimization as Circuit Complexity, Phys. Rev. Lett. 123, 011601 (2019).

[58] S. A. Hartnoll, Lectures on holographic methods for condensed matter physics, Classical Quantum Gravity 26, 224002 (2009).

[59] C. P. Herzog, Lectures on holographic superfluidity and superconductivity, J. Phys. A 42, 343001 (2009).

[60] S. Sachdev, What can gauge-gravity duality teach us about condensed matter physics?, Annu. Rev. Condens. Matter Phys. 3, 9 (2012).

[61] Y. Liu, K. Schalm, Y. W. Sun, and J. Zaanen, Lattice potentials and fermions in holographic non Fermi-Liquids: Hybridizing local quantum criticality, J. High Energy Phys. 10 (2012) 036.

[62] Y. Ling, C. Niu, J. P. Wu, Z. Y. Xian, and H. b. Zhang, Holographic fermionic liquid with lattices, J. High Energy Phys. 07 (2013) 045.

[63] Y. Ling, P. Liu, C. Niu, J. P. Wu, and Z. Y. Xian, Holographic fermionic system with dipole coupling on $Q$-lattice, J. High Energy Phys. 12 (2014) 149.

[64] M. Reza Mohammadi Mozaffar, A. Mollabashi, and F. Omidi, Non-local probes in holographic theories with momentum relaxation, J. High Energy Phys. 10 (2016) 135.

[65] S. Cremonini, L. Li, and J. Ren, Holographic fermions in striped phases, J. High Energy Phys. 12 (2018) 080.

[66] S. Cremonini, L. Li, and J. Ren, Spectral weight suppression and fermi arc-like features with strong holographic lattices, J. High Energy Phys. 09 (2019) 014.

[67] G. T. Horowitz, J. E. Santos, and D. Tong, Optical conductivity with holographic lattices, J. High Energy Phys. 07 (2012) 168.

[68] R. A. Davison, Momentum relaxation in holographic massive gravity, Phys. Rev. D 88, 086003 (2013).

[69] M. Blake and D. Tong, Universal resistivity from holographic massive gravity, Phys. Rev. D 88, 106004 (2013).

[70] Y. Ling, C. Niu, J. P. Wu, and Z. Y. Xian, Holographic lattice in Einstein-Maxwell-Dilaton gravity, J. High Energy Phys. 11 (2013) 006.

[71] A. Donos and J.P. Gauntlett, Holographic Q-lattices, J. High Energy Phys. 04 (2014) 040.

[72] T. Andrade and B. Withers, A simple holographic model of momentum relaxation, J. High Energy Phys. 05 (2014) 101.

[73] A. Donos and J. P. Gauntlett, The thermoelectric properties of inhomogeneous holographic lattices, J. High Energy Phys. 01 (2015) 035.

[74] R. A. Davison and B. Goutraux, Dissecting holographic conductivities, J. High Energy Phys. 09 (2015) 090.
[75] C. de Rham, G. Gabadadze, and A. J. Tolley, Resummation of Massive Gravity, Phys. Rev. Lett. 106, 231101 (2011).

[76] S. A. Hartnoll, Theory of universal incoherent metallic transport, Nat. Phys. 11, 54 (2015),

[77] J. Maldacena, S. H. Shenker, and D. Stanford, A bound on chaos, J. High Energy Phys. 08 (2016) 106.

[78] S. H. Shenker and D. Stanford, Black holes and the butterfly effect, J. High Energy Phys. 03 (2014) 067.

[79] D. A. Roberts, D. Stanford, and L. Susskind, Localized shocks, J. High Energy Phys. 03 (2015) 051.

[80] C. Armendariz-Picon, T. Damour, and V.F. Mukhanov, k-Inflation, Phys. Lett. B 458, 209 (1999).

[81] M. Baggioli and O. Pujolas, Electron-Phonon Interactions, Metal-Insulator Transitions, and Holographic Massive Gravity, Phys. Rev. Lett. 114, 251602 (2015).

[82] A. Cisterna, M. Hassaine, J. Oliva, and M. Rinaldi, Axionic black branes in the $k$-essence sector of the Horndeski model, Phys. Rev. D 96, 124033 (2017).

[83] Y. T. Zhou, X. M. Kuang, Y.Z. Li, and J. P. Wu, Holographic subregion complexity under a thermal quench in an Einstein-Maxwell-axion theory with momentum relaxation, Phys. Rev. D 101, 106024 (2020).

[84] G. W. Horndeski, Second-order scalar-tensor field equations in a four-dimensional space, Int. J. Theor. Phys. 10, 363 (1974).

[85] T. Kobayashi, M. Yamaguchi, and J. Yokoyama, Generalized G-inflation: Inflation with the most general secondorder field equations, Prog. Theor. Phys. 126, 511 (2011).

[86] R. C. Myers, Stress tensors and Casimir energies in the AdS/CFT correspondence, Phys. Rev. D 60, 046002 (1999).

[87] L. Q. Fang and X. M. Kuang, Holographic heat engine with momentum relaxation, Sci. China Phys. Mech. Astron. 61, 080421 (2018).

[88] J. W. York, Jr., Role of Conformal Three Geometry in the Dynamics of Gravitation, Phys. Rev. Lett. 28, 1082 (1972).

[89] G. W. Gibbons and S. W. Hawking, Action integrals and partition functions in quantum gravity, Phys. Rev. D 15, 2752 (1977).

[90] K. Goto, H. Marrochio, R. C. Myers, L. Queimada, and B. Yoshida, Holographic complexity equals which action?, J. High Energy Phys. 02 (2019) 160.

[91] H. Casini, M. Huerta, and R. C. Myers, Towards a derivation of holographic entanglement entropy, J. High Energy Phys. 05 (2011) 036.

[92] B. Goutraux, Charge transport in holography with momentum dissipation, J. High Energy Phys. 04 (2014) 181.

[93] P. Hayden and J. Preskill, Black holes as mirrors: Quantum information in random subsystems, J. High Energy Phys. 09 (2007) 120.

[94] K. Damle and S. Sachdev, Nonzero-temperature transport near quantum critical points, Phys. Rev. B 56, 8714 (1997); Lieb-Robinson and the Butterfly Effect, Phys. Rev. Lett. 117, 091602 (2016).

[95] S. A. Hartnoll, A. Lucas, and S. Sachdev, Holographic quantum matter, arXiv:1612.07324.

[96] M. Blake, Universal Charge Diffusion and the Butterfly Effect in Holographic Theories, Phys. Rev. Lett. 117, 091601 (2016). 
[97] P. Kovtun, D. T. Son, and A. O. Starinets, Viscosity in Strongly Interacting Quantum Field Theories from Black Hole Physics, Phys. Rev. Lett. 94, 111601 (2005).

[98] M. Blake, R. A. Davison, and S. Sachdev, Thermal diffusivity and chaos in metals without quasiparticles, Phys. Rev. D 96, 106008 (2017).

[99] H. S. Jeong, K. Y. Kim, and Y. W. Sun, Bound of diffusion constants from pole-skipping points: Spontaneous symmetry breaking and magnetic field, J. High Energy Phys. 07 (2021) 105.

[100] N. Iqbal and H. Liu, Universality of the hydrodynamic limit in AdS/CFT and the membrane paradigm, Phys. Rev. D 79, 025023 (2009).

[101] X. H. Feng and H. Lu, Butterfly velocity bound and reverse isoperimetric inequality, Phys. Rev. D 95, 066001 (2017).

[102] M. Baggioli, B. Padhi, P. W. Phillips, and C. Setty, Conjecture on the butterfly velocity across a quantum phase transition, J. High Energy Phys. 07 (2018) 049.

[103] S. Q. Hu and X. M. Kuang, Holographic heat engine in Horndeski model with the $k$-essence sector, Sci. China Phys. Mech. Astron. 62, 60411 (2019).
[104] Y. Ling, Z. Y. Xian, and Z. Zhou, Holographic shear viscosity in hyperscaling violating theories without translational invariance, J. High Energy Phys. 11 (2016) 007.

[105] M. Alishahiha, A. Faraji Astaneh, M. R. Mohammadi Mozaffar, and A. Mollabashi, Complexity growth with Lifshitz scaling and hyperscaling violation, J. High Energy Phys. 07 (2018) 042.

[106] A. B. Zamolodchikov, Expectation value of composite field $T$ anti- $T$ in two-dimensional quantum field theory, arXiv:hep-th/0401146.

[107] L. McGough, M. Mezei, and H. Verlinde, Moving the CFT into the bulk with $T \bar{T}$, J. High Energy Phys. 04 (2018) 010.

[108] A. Akhavan, M. Alishahiha, A. Naseh, and H. Zolfi, Complexity and behind the horizon cut off, J. High Energy Phys. 12 (2018) 090.

[109] M. Alishahiha and A. Faraji Astaneh, Complexity of hyperscaling violating theories at finite cutoff, Phys. Rev. D 100, 086004 (2019).

[110] S. S. Hashemi, G. Jafari, A. Naseh, and H. Zolfi, More on complexity in finite cut off geometry, Phys. Lett. B 797, 134898 (2019). 